\title{
High iodine adsorption by polyethyleneimine impregnated
}

\section{nanosilica sorbents}

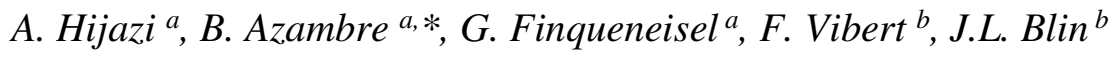 \\ ${ }^{\text {a }}$ Laboratoire de Chimie et Physique-Approche Multi-Echelle des Milieux Complexes (LCP- \\ A2MC- EA n²362), Institut Jean-Barriol FR2843 CNRS, Université de Lorraine, Rue Victor \\ Demange, 57500 Saint-Avold, France. \\ ${ }^{\text {b }}$ Laboratoire Lorrain de Chimie Moléculaire (L2CM), UMR CNRS 7053, Université de \\ Lorraine, Faculté des sciences et technologies - Boulevard des Aiguillettes BP 239, 54506 \\ Vandoeuvre les Nancy
}

*Corresponding Author. E-mail address: bruno.azambre@univ-lorraine.fr

KEYWORDS: SBA-15, mesoporous, adsorption, nuclear application, charge-transfer complex, halogen bonding

\begin{abstract}
:
In this study, several nanoporous silica of SBA-15 and Aerosil types were impregnated with branched polyethyleneimine (PEI) with the aim to evaluate their iodine adsorption performance. First, the influence of the PEI content of the sorbents on their textural, structural, thermal and chemical characteristics, was studied by different techniques including $\mathrm{N}_{2}$ porosimetry at $77 \mathrm{~K}$, SAXS, FTIR/ATR, elemental analysis, and TGA. Then, $\mathrm{I}_{2}$ adsorption capacities were determined in liquid phase (cyclohexane) at $20^{\circ} \mathrm{C}$, or during static or dynamic gas-phase tests, performed at 60 and $100^{\circ} \mathrm{C}$, respectively. An exceptionally high adsorption capacity superior to $2 \mathrm{~g} / \mathrm{g}$ in the 20 $100{ }^{\circ} \mathrm{C}$ range was determined for the SBA-15 sorbent with $38 \mathrm{wt} \%$ PEI (corresponding to $\mathrm{N}$ content of $12.3 \mathrm{wt} \%$ ). The adsorption capacities of the sorbents increase almost linearly with the
\end{abstract}


$\mathrm{N}$ content below this threshold, whereas the presence of excess PEI in the pores or at the pore mouth reduces the accessibility of $I_{2}$ molecules to the most buried nitrogen sites. The interaction mechanisms of iodine with amine-containing molecules were investigated both from experiments carried out in solution and from the after-test characterization of the spent solids by DR-UV-Vis, FTIR/ATR, Raman spectroscopies and iodine desorption monitored by TGA. This allowed shedding light on the nature of charge-transfer complexes and the formation of ionic and neutral iodine ad-species during iodine adsorption.

\section{Introduction}

The different forms of radioactive iodine $\left({ }^{131} \mathrm{I}\right.$ and $\left.{ }^{129} \mathrm{I}\right)$ are among the most important radiotoxic species which could be released in case of major nuclear accident [1]. Owing to their high volatility and radiological impacts on human beings and the environment, the capture of radioiodine species (namely $\mathrm{I}_{2}$ and organic iodides such as $\mathrm{CH}_{3} \mathrm{I}$ ) has become a major issue for safe nuclear energy [2]. Different mitigation means (known also as "wet" and "dry" filtration systems) are currently examined for iodine [3,4]. Although iodine aerosols $\left(\mathrm{I}_{\mathrm{x}} \mathrm{O}_{\mathrm{y}}, \mathrm{CsI}, \ldots\right)$ could be efficiently trapped by wet scrubbers or sand beds, these types of filters are not efficient for the volatile forms of iodine. By contrast, porous sorbents, such as silver zeolites and impregnated activated carbons are known for a long time to have suitable properties for the trapping of $I_{2}$ and $\mathrm{CH}_{3} \mathrm{I}$, at least under certain conditions, especially zeolites [5]. In view of a possible implementation on the venting line of the containment (i.e. Filter Containment Venting System (FCVS) application in French nuclear power plants), candidate sorbents have to withstand harsh conditions of temperature, pressure, irradiation and the presence of other chemicals while still 
retaining iodine species [6]. Since the Fukushima accident, the behavior of silver zeolites is still currently revisited with this aim [6-9].

Beside the works carried out onto these industrial sorbents, the quest for novel sorbents have also attracted a significant interest in the last years [10,11]. For instance, molecular or hybrid sorbents based on embedded polymers in inorganic matrices, or the reverse, were widely used these last years in a variety of environmental applications [12-15]. In the field of iodine capture, very high adsorption capacities for $\mathrm{I}_{2}$ (up to $\approx 2-3 \mathrm{~g}$ of $\mathrm{I}_{2}$ per $\mathrm{g}$ of sorbent) were sometimes reported for specific molecular or hybrid materials belonging to the classes of Metal and Covalent Organic Frameworks (MOFs [10] and COFs [12], respectively) or Porous Organic Polymers [13]. Their adsorption properties were recently discussed and emphasized in some reviews $[4,14,15]$.

On the other hand, it is rather surprising that mesoporous silica-based materials were only scarcely studied for iodine adsorption [16,17]. However, mesostructured silicas constitute a class of ordered materials presenting high specific surface areas $\left(\approx 500-1000 \mathrm{~m}^{2} / \mathrm{g}\right)$ and a large variety of structures (ex: SBA-15, MCM-41, KIT-6...) [18]. Their tunable pore size in the mesopore range $(2-10 \mathrm{~nm})$ confers them potential advantages over microporous zeolites [19], such as a better accessibility for iodine and in principle a lesser sensitivity to pore blockage by precipitates or adsorbed species. Moreover, the possibility of functionalization with organic groups or doping with metals can be exploited to increase the affinity of silica for iodine [20].

In that respect, the incorporation of silver and bismuth in mesoporous silicas (SBA-15 and MCM-41) was investigated because these elements could form stable AgI [16] and $\mathrm{BiI}_{3}$ [17] precipitates by reaction with iodine. For bismuth, a maximal adsorption capacity of $540 \mathrm{mg}(\mathrm{I}) / \mathrm{g}$ was reached at $200{ }^{\circ} \mathrm{C}$ after a previous surface modification with thiol groups and a thermal treatment to form bismuth sulfide [17]. By contrast, the impregnation of silver nitrate, even in 
high amounts, did not lead to an improvement of adsorption performances in comparison with silver-exchanged zeolites, especially for $\mathrm{CH}_{3} \mathrm{I}$ [20]. On the other hand, the effect of surface functionalization by organic groups was only investigated by us recently. The grafting of aminosilane reagents on the silanol groups of SBA-15 yielded to a strong enhancement of the $\mathrm{I}_{2}$ adsorption capacity (up to $600 \mathrm{mg} / \mathrm{g}$ at $25^{\circ} \mathrm{C}$ ) in comparison with the parent (non-functionalized) silica $(<50 \mathrm{mg} / \mathrm{g})$ [20]. In that respect, the good performances of this amino-functionalized SBA15 could be somewhat compared to those of MOF's bearing $\mathrm{NH}_{2}$-functionalized ligands or triethylenediamine (TEDA)-impregnated charcoals [21]. As reported already long time ago for homogeneous systems, they have probably for origin the formation of charge-transfer complexes between amine groups (the donor) and molecular iodine (the acceptor) [22].

We chose to further explore this way by investigating other systems composed of mesoporous silicas and embedded amine species, by reporting here a study on polyethyleneimine(PEI)impregnated SBA-15 materials. PEI is a polymer with repeating units composed of one amine group and one aliphatic $-\mathrm{CH}_{2}-\mathrm{CH}_{2}-$ spacer. Linear polyethyleneimines contain all secondary amines, in contrast to branched PEIs which contain primary, secondary and tertiary amino groups [23]. By impregnation on mesoporous silica, it was shown that the obtained sorbents displayed excellent adsorption capacities for $\mathrm{CO}_{2}$ from air, as well as good stability of adsorption performances over several regeneration cycles $[24,25]$. In this study, our assumption was that the high density of amine groups in PEI could yield a lot of active sites for iodine sorption. The hexagonal SBA-15 structure, with cylindrical large pores of size 6-8 nm, was selected because of its robustness and hydrothermal stability compared to other forms of nanoporous silicas. In this paper, several PEI-SBA-15 sorbents containing different amounts of polyethyleneimine, previously grafted or not with aminosilane, were first characterized by different techniques 
(SAXS, $\mathrm{N}_{2}$ sorptiometry, FTIR/ATR). Then, their adsorption properties for iodine were investigated either in cyclohexane solvent at $20{ }^{\circ} \mathrm{C}$ or in gas-phase test at $100{ }^{\circ} \mathrm{C}$. Finally, a mechanism was proposed to explain the observed trends by exploring the interactions existing between PEI and iodine using DR-UV-Vis and Raman spectroscopies.

\section{Experimental Section}

\subsection{Materials and Syntheses}

Reagents. For SBA-15 synthesis, Tetraethylorthosilicate (TEOS) (purity >98\%) and PEO-PPOPEO Pluronic polymer (P123, Mn 5800) were purchased from Sigma-Aldrich. The branched polyethyleneimine (PEI) (Mw 600, 99\%) used for the impregnation of SBA-15 was supplied by Alfa Aesar (a typical structure is provided on Figure S1). Methanol solvent (99.8\%, extra dry over Molecular Sieves, AcroSeal), and N-(2-aminoethyl)-3-aminopropyl trimethoxy silane (AEAPTMS) (purity 97\%) was purchased from Acros Organics. Finally, the iodine pellets (ACS reagent, $\geq 99.8 \%$, solid) used in the PEI-iodine interaction and adsorption tests are provided by Sigma-Aldrich.

Synthesis of SBA-15. The parent SBA-15 was synthesized by the cooperative self-assembly mechanism using a protocol adapted from Zhao et al. [19] with a starting molar ratio: 1.0 TEOS: 0.017 P123: $5.5 \mathrm{HCl}: 172 \mathrm{H}_{2} \mathrm{O}$. First, $16.8 \mathrm{~g}$ of $\mathrm{P} 123$ were dissolved at $40{ }^{\circ} \mathrm{C}$ in an acidic solution composed of $526 \mathrm{~mL}$ of distilled water and $78 \mathrm{~mL}$ of $\mathrm{HCl} \approx 12 \mathrm{M}$ (36 wt $\%$ ). Then, 35.5 g TEOS were slowly added and a continuous stirring $(550 \mathrm{rpm})$ was maintained at $40{ }^{\circ} \mathrm{C}$ during $24 \mathrm{~h}$ to control the organization and growth of the mesophase during the ripening step. The temperature was then raised to $90{ }^{\circ} \mathrm{C}$ and the material was further aged under reflux for $48 \mathrm{~h}$. After cooling down to room temperature, the synthesized product was collected by Büchner filtration and washed several times with distilled water, then kept overnight to dry at $80{ }^{\circ} \mathrm{C}$. 
Finally, the mesoporous SBA-15 material was obtained after calcination at $550{ }^{\circ} \mathrm{C}$ for $6 \mathrm{~h}$ (static air, heating rate $=2{ }^{\circ} \mathrm{C} / \mathrm{min}$ ).

Grafting of SBA-15 by AE-APTMS. $1 \mathrm{~g}$ of the calcined SBA-15 material was first boiled in distilled water at $100{ }^{\circ} \mathrm{C}$ for 2 hours in order to increase the concentration of surface silanol groups. Then, the mesoporous silica was refluxed in presence of a toluene solution at $90{ }^{\circ} \mathrm{C}$ containing $17 \mathrm{wt} \%$ of amino-ethyl amino-propyl tri-methoxy silane (AE-APTMS). The adsorbent was finally named AE-APTMS-SBA-15.

Impregnation of nanosilicas by PEI. The PEI-modified SBA-15 samples were prepared by a wet impregnation method. In a typical procedure, the desired amount of PEI was dissolved in $4 \mathrm{~mL}$ of methanol, then $1 \mathrm{~g}$ of the calcined SBA-15 was added. The slurry obtained was continuously stirred for $15 \mathrm{~min}$, then dried overnight under vacuum at $80^{\circ} \mathrm{C}$. A commercial fumed silica bearing no internal porosity (Aerosil200, Sigma-Aldrich, silica content $>99.8 \%$ ) was also impregnated by a similar procedure for comparison. The as-prepared samples were named as xPEI-sorbent, where $x$ represents the mass of PEI (in g/g of SBA-15) used for impregnation: 0.25PEI-SBA-15, 0.75PEI-SBA-15， 1.6PEI-SBA-15， 0.25PEI-AE-APTMS-SBA-15, and 0.25PEI-Aerosil.

\subsection{Characterization of fresh and spent adsorbents}

Elemental analyses were performed at the Service d'Analyses Elementaires at University of Lorraine (France).

Small Angle X-ray Scattering (SAXS). SAXS measurements were performed using a SAXSess $\mathrm{mc}^{2}$ instrument (Anton Paar). Samples placed between two Kapton ${ }^{\circledR}$ sheets were exposed to a $\mathrm{CuK} \alpha$ radiation $(\lambda=0.1542 \mathrm{~nm})$. Scattered beams were registered by a CCD detector placed at $309 \mathrm{~mm}$ away from the sample. The resultant 2D image was integrated by SAXSQuant software 
(Anton Paar) into one-dimensional variation between the scattering intensities $\mathrm{I}(\mathrm{q})$ and the scattering vector $q=(4 \pi / \lambda) \sin (\theta)$, where $2 \theta$ is the total scattering angle, such that all intensities were normalized according to an attenuated primary beam $(q=0)$. The data were finally corrected for the background scattering from Kapton ${ }^{\circledR}$. Lattice parameters were determined from the scattering vector $(\mathrm{q})$ such that: the $\mathrm{d}$-spacing $\left(\mathrm{d}_{100}\right)=2 \pi / \mathrm{q}$, and the cell parameter $\left(\mathrm{a}_{0}\right)=$ $2 \mathrm{~d}_{100} / \sqrt{3}$.

$N_{2}$ porosimetry at $77 \mathrm{~K}$. Nitrogen adsorption-desorption isotherms at $77 \mathrm{~K}$ were measured over relative pressures range 0.01-0.995 using an Autosorb IQ sorptiometer from Quantachrome. A thermal treatment at $80{ }^{\circ} \mathrm{C}$ for $4 \mathrm{~h}$ (heating rate $=5{ }^{\circ} \mathrm{C} / \mathrm{min}$ ) under vacuum was performed to remove most of adsorbed impurities and humidity. BET (Brunauer-Emmett-Teller) theory was used to determine the specific surface areas of the sorbents in the range $\left(0.05 \leq \mathrm{P} / \mathrm{P}_{0} \leq 0.2\right)$. The pore size distribution, pore volumes, and the pore diameters were determined by BJH (BarrettJoyner-Halenda) model applied to the desorption branch of the isotherm. The micropores volume and area were obtained by $t$-plot method using de Boer's model from the equation of thickness $(t$ $=\mathrm{a}\left[\frac{1}{\ln \left(\frac{P 0}{P}\right)}\right]^{1 / \mathrm{b}}$ such that $\mathrm{a}=6.0533$ and $\left.\mathrm{b}=3.0\right)$.

Because the amounts of PEI in some of the prepared materials were noticeably high, porosimetric data reported in Table 1 and Fig. 3 were normalized according to the $\mathrm{SiO}_{2}$ content in each PEI-impregnated materials. Hence, it allows to describe the evolution of textural properties of PEI-impregnated SBA-15 sorbents on a PEI-free basis.

Thermogravimetric Analysis (TGA). TG measurements were carried out in order to gain insights both on the thermal stability of the impregnated PEI and the stability of adsorbed iodine species. The mass losses underwent by 10-12 mg samples as function of temperature were recorded 
under nitrogen (flow rate $=60 \mathrm{~mL} / \mathrm{min}$ ) using a $\mathrm{TG}$ apparatus from Setaram Instrumentation (KEP Technologies), of LabSys evo model. The samples were first kept at $30{ }^{\circ} \mathrm{C}$, then a thermal treatment was started from $30{ }^{\circ} \mathrm{C}$ up to $900{ }^{\circ} \mathrm{C}$ at a heating rate of $10^{\circ} \mathrm{C} / \mathrm{min}$.

DRS-UV-Vis spectroscopy. Diffuse reflectance UV-Vis spectra of PEI-impregnated samples before and after iodine tests were collected on Cary $4000 \mathrm{UV}$-Vis spectrometer from Agilent Technologies equipped with a double monochromator and DRA 900 integrating sphere. DR-UVVis spectra were registered between 200 and $800 \mathrm{~nm}$, with a scan rate of $600 \mathrm{~nm} / \mathrm{min}$ and a resolution of $2 \mathrm{~nm}$, with the parent SBA-15 used as a reference sample.

FTIR/ATR spectroscopy. ATR-IR spectra of PEI-impregnated samples before and after iodine tests were collected using a Bruker Alpha spectrometer equipped with Alpha P ATR accessory and diamond crystal. The measurements were done between 400 and $4000 \mathrm{~cm}^{-1}$ with a resolution of $4 \mathrm{~cm}^{-1}$ and co-addition of 32 scans.

Raman spectroscopy. Raman spectra of iodine species were collected between 120 and $500 \mathrm{~cm}^{-1}$ on a BWTek-i-Raman portable spectrometer using a $785 \mathrm{~nm}$ laser. The laser power and time collection were optimized for each sample in order to optimize the signal while preserving the samples from burning locally.

\subsection{Iodine adsorption tests}

Liquid-phase experiments. The adsorption properties of the prepared sorbents were investigated using molecular iodine $\left(\mathrm{I}_{2}\right)$ solutions prepared in cyclohexane solvent. For most of the tests with molecular iodine, $75 \mathrm{mg}$ of sorbent was contacted at $20 \pm 5^{\circ} \mathrm{C}$ in the dark with $50 \mathrm{~mL}$ of a 3000 ppm solution made from $\mathrm{I}_{2}$ pellets (Alfa Aesar, 99.5\%). This concentration was checked to be sufficient to reach the maximal adsorption capacity of each sorbent. Determination of adsorbed amounts at equilibrium was done by UV-visible spectrophotometry using a double-beam Cary 
4000 UV-Vis spectrophotometer (Agilent Technologies) and $1 \mathrm{~cm}$ path length quartz cuvettes. Absorbance values of the initial solution and after $48 \mathrm{~h}$ equilibrium were measured at $523 \mathrm{~nm}$ and the adsorptions capacities (in $\mathrm{mg} / \mathrm{g}$ ) were calculated using the following formula:

$$
\mathrm{Q}_{\mathrm{I} 2}=\frac{A 0-A f}{3.6244 \times \text { mass of adsorbent }} \times \mathrm{V}_{\text {solution }}
$$

Gas-phase experiments. In the first series of experiments, the iodine uptake was determined for all the sorbents by gravimetry under static conditions. $100 \mathrm{mg}$ of each sorbent were spread onto an open glass plate. The plates were then placed in the first stage of a glass sealed desiccator containing $4 \mathrm{~g}$ of iodine pellets at the bottom and the whole set-up was introduced in an oven at $60{ }^{\circ} \mathrm{C}$ for $12 \mathrm{~h}$. The samples were then removed from the adsorption vessel and kept under ventilation for $7 \mathrm{~h}$ to eliminate the weakest fraction of adsorbed iodine. Finally, they were weighted again to determine the adsorbed mass of $\mathrm{I}_{2}$. The iodine uptake was calculated using: $\mathrm{Q}\left(\mathrm{I}_{2}\right)=\left(\mathrm{m}_{\text {final }}-\mathrm{m}_{\text {initial }}\right) / \mathrm{m}_{\text {initial. }}$. The equilibrium gas-phase concentration in our experiments was systematically deduced from the consumption of iodine pellets (introduced in large excess) during blank experiments and also experiments done in presence of the adsorbents. As the tendency of the sample to adsorb iodine increases, more iodine is sublimated in order to compensate the part of iodine which is adsorbed (according to Le Châtelier's Law), but overall, the equilibrium pressure was found to be approximately the same in each experiment: $\mathrm{P}_{\mathrm{I} 2}=$ $356+/-20 \mathrm{~Pa}$ at $60^{\circ} \mathrm{C}$ which is rather close to the value corresponding to the T-P 12 curve [30].

The iodine adsorption capacity was also determined for some selected sorbents using another experimental setup working under conditions closer to those met in a severe nuclear accident (flowing gases, higher temperature). An extended description of this setup is given in Ref. [6] Briefly, $200 \mathrm{mg}$ of sorbent (previously outgassed at $150{ }^{\circ} \mathrm{C}$ under $\mathrm{Ar}$ ) were placed inside a glass reactor and exposed to an Argon flow $(100 \mathrm{~mL} / \mathrm{min})$. Once the pre-treatment was completed, the 
temperature was cooled down to $100{ }^{\circ} \mathrm{C}$ and the sorbent was exposed to an Ar flow containing a reproducible concentration of $1240 \mathrm{ppm}$ iodine. The concentration of iodine downstream the sorbent was continuously monitored by UV-Vis spectroscopy from absorbance measurements taken at $523 \mathrm{~nm}$. Adsorption capacities at breakthrough and saturation of the bed were calculated from the integration of the $\mathrm{I}_{2}$ breakthrough curve [6].

\section{Results and Discussion}

\subsection{Characterization of the prepared sorbents}

Table 1. Chemical, structural, and textural properties of SBA-15 and PEI-impregnated samples

\begin{tabular}{lllllllll}
\hline Sorbent & $\begin{array}{l}\text { \%PEI } \\
(\mathrm{wt} \%)\end{array}$ & $\mathrm{d}_{100}(\mathrm{~nm})$ & $\mathrm{a}_{0}(\mathrm{~nm})$ & $\begin{array}{l}\mathrm{S}_{\mathrm{BET}} \\
\left(\mathrm{m}^{2} / \mathrm{g}\right)\end{array}$ & $\begin{array}{l}\mathrm{S}_{\text {micro }} \\
\left(\mathrm{m}^{2} / \mathrm{g}\right)\end{array}$ & $\begin{array}{l}\mathrm{V}_{\text {micro }} \\
\left(\mathrm{cm}^{3} / \mathrm{g}\right)\end{array}$ & $\begin{array}{l}\mathrm{V}_{\text {pore }} \\
\left(\mathrm{cm}^{3} / \mathrm{g}\right)\end{array}$ & $\begin{array}{l}\mathrm{D}_{\text {pore }} \\
(\mathrm{nm})\end{array}$ \\
\hline SBA-15 & 0 & 9.7 & 11.2 & 725 & 187 & 0.076 & 0.96 & 6.8 \\
0.25PEI- SBA-15 & 19 & 9.7 & 11.2 & 309 & 0 & 0 & 0.65 & 6.3 \\
0.75PEI- SBA-15 & 38 & 9.7 & 11.2 & 40 & 0 & 0 & 0.13 & $6.3,4$ \\
1.6PEI- SBA-15 & 51 & 9.7 & 11.2 & 0 & 0 & 0 & $<0.03$ & - \\
AE-APTMS-PEI- SBA-15 & 0 & 9.7 & 11.2 & 286 & 0 & 0 & 0.68 & 5.8 \\
0.25PEI-AE-APTMS- SBA-15 & 11 & 9.7 & 11.2 & 107 & 0 & 0 & 0.25 & $5.8,3.7$ \\
Aerosil & 0 & - & - & 177 & 0 & 0 & 0.35 & - \\
0.25PEI-Aerosil & 19 & - & - & 85 & 0 & 0 & 0.31 & - \\
\hline
\end{tabular}

Chemical and thermal analyses

Elemental analyses. For the series of impregnated SBA-15 samples constituted by 0.25PEI-SBA15, 0.75PEI-SBA-15 and 1.6PEI-SBA-15, the PEI loadings are 19, 38 and $51 \mathrm{wt} \%$, respectively (as deduced from elemental analyses provided in Table S1). From theoretical calculations taking a pore volume of $0.96 \mathrm{~cm}^{3} / \mathrm{g}$ for the parent SBA-15, the maximal PEI content that could be allocated in the pores should be $0.988 \mathrm{~g}$, which corresponds to a PEI content of $49.7 \mathrm{wt} \%$. In this study, the 1.6PEI-SBA-15 sample with the highest PEI content (51 wt\%) had a sticky aspect (paste-like) and noticeably differed from the powdered aspect of the others. Hence, it is expected that a significant fraction of PEI is located on the external surface of SBA-15 particles. It is worth noting that the same behavior was observed for the pyrogenic Aerosil silica but at a much 
lower PEI loading (19 wt\%). In that case, the paste-like aspect of this sample originated from the absence of internal porosity and the presence of PEI on the external surface or in the empty voids between the silica nanoparticles.

Another SBA-15 sample was post-grafted with an aminosilane (AE-APTMS), in order the further examine the possibility of achieving an improved dispersion of PEI in the pores due to the chemical compatibility between these two molecules. Once the SBA-15 was post-grafted with AE-APTMS, the $\mathrm{N}$ content was equal to $5.6 \mathrm{wt} \%$ and the impregnation of PEI made it further increased to $9.3 \mathrm{wt} \%$. This increase corresponds to a PEI loading of approximately 11 wt $\%$.

Infrared spectroscopy. All FTIR/ATR spectra of SBA-15 samples displayed the IR peaks corresponding to symmetric $\left(800 \mathrm{~cm}^{-1}\right)$ and asymmetric stretching vibrations $\left(1045 \mathrm{~cm}^{-1}\right)$ of $\mathrm{Si}-$ O-Si silica framework as well as Si-O-Si bending vibration at $435 \mathrm{~cm}^{-1}$. The presence of silanols can also be detected ca $950 \mathrm{~cm}^{-1}$ (as a shoulder of the main band) [26].

In the case of pure branched PEI (in liquid state, Fig. 1, black line), the stretching vibrations of secondary and primary amines are observed at 3350, 3272 (main), $3180 \mathrm{~cm}^{-1}$ while the deformation bands are at 1650 (shoulder) and $1590 \mathrm{~cm}^{-1}$. The $\mathrm{C}-\mathrm{N}$ stretches are found at 1121 and $1046 \mathrm{~cm}^{-1}$ whereas the vibrations belonging to ethylene moieties are located at 2929,2884 , $2806 \mathrm{~cm}^{-1}\left(\mathrm{CH}_{2}\right.$ stretch) and $1453 \mathrm{~cm}^{-1}\left(\mathrm{CH}_{2}\right.$ bend). By comparison, the ATR spectra of PEIimpregnated nanosilicas displayed the same vibrations (besides the bands related to silica framework, Fig. 1), but most of C-H or N-H related-bands were more or less broadened or frequency-shifted (by less than $20 \mathrm{~cm}^{-1}$ ) due to the occurrence of specific interactions between PEI and the surface. Those spectral perturbations were found to be more important for the smaller PEI contents and tend to be less visible as the PEI content increased (e.g. for 1.6PEI- 
SBA-15). Hence, they should probably involve the fraction of PEI molecules located in the internal porosity or in direct interaction with the surface. This type of PEI molecules may experience for instance some confinement in the mesopores or interact by H-bonding with silanol species or adsorbed water. The presence of silanol groups was reported to serve as reaction sites for amine grafting, enhancing the dispersion of PEI along the pores and also $\mathrm{CO}_{2}$ pore diffusion [27]. Moreover, the presence of adsorbed water or acidic silanol species at silica surface is expected to induce some protonation of some amine groups of PEI in their vicinity, forming their corresponding ammonium salts. As shown on Fig. 1 (grey line, $\mathrm{PEI}+\mathrm{H}_{2} \mathrm{O}$ ), the spectrum obtained by mixing PEI and water and subtracting the contribution of water displays some similarities with the spectra of PEI-impregnated nanosilicas at low PEI loadings, indicating the protonation or interaction of some amine groups with the surface. However, as the PEI content increases, layers of PEI molecules progressively accumulate on the external surface and the IR spectrum of such molecules should be more resembling to that of liquid PEI.

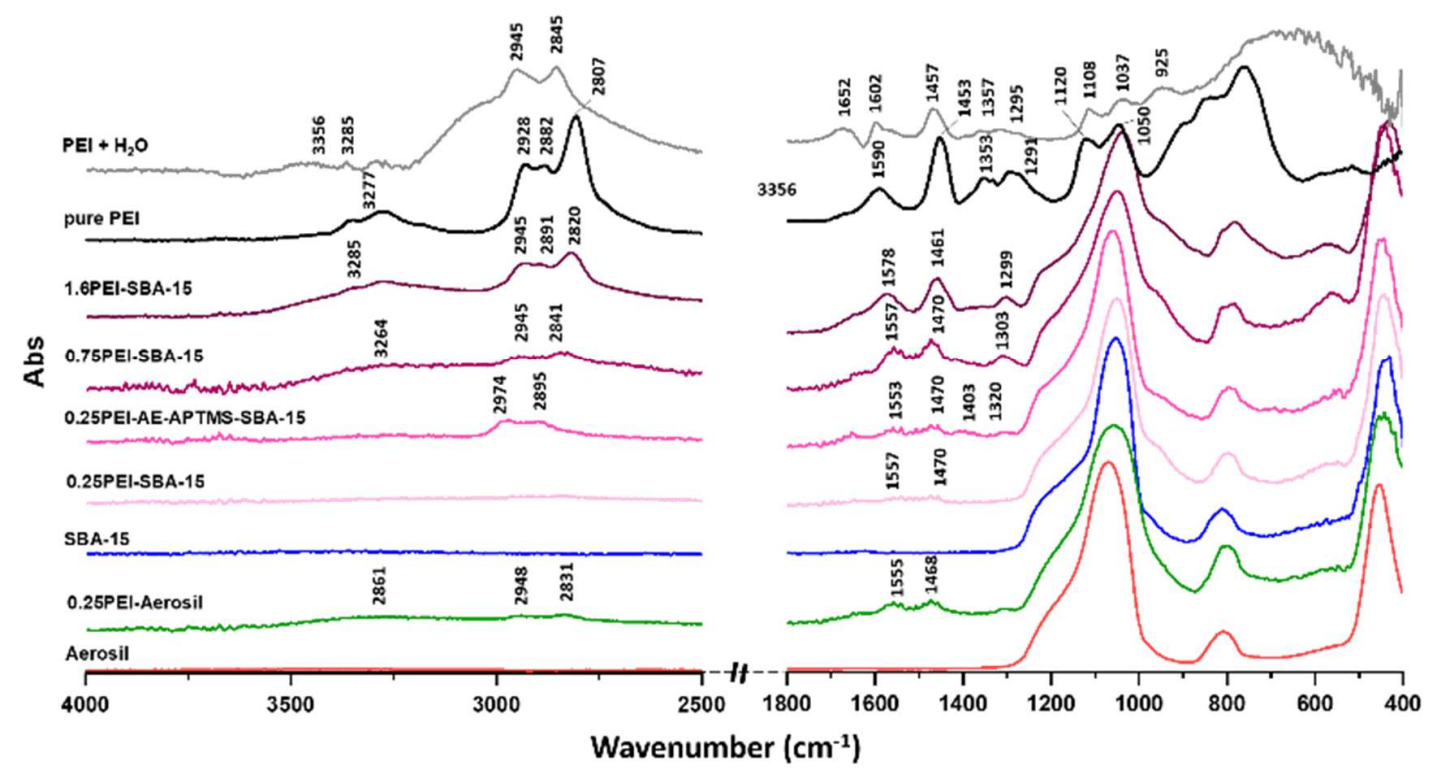


Fig. 1. ATR-Infrared spectra of the as-prepared PEI-impregnated samples and PEI reference

Thermogravimetry. The thermogravimetric analyses (Fig. 2, black line) of the liquid PEI reference under $\mathrm{N}_{2}$ atmosphere $\left(\mathrm{v}=10{ }^{\circ} \mathrm{C} / \mathrm{min}\right)$ shows that its thermal degradation is slowly initiated at $200{ }^{\circ} \mathrm{C}$ while most of the mass loss occurs between 320 and $380{ }^{\circ} \mathrm{C}$, resulting to a sharp and symmetric peak observed on DTG signal $\left(\mathrm{DTG}_{\max }=353{ }^{\circ} \mathrm{C}\right)$. According to low molecular weight $\left(\mathrm{Mw}=600 \mathrm{~g} \cdot \mathrm{mol}^{-1}\right)$ of the branched PEI used, this seems to be more indicative of an evaporation process rather than decomposition.

By contrast, PEI-impregnated samples exhibited several distinct mass losses (Fig. 2). The first loss observed at low temperature $\left(\mathrm{DTG}_{\max }=83-112^{\circ} \mathrm{C}\right.$ ) is assigned to $\mathrm{H}_{2} \mathrm{O}$ and/or $\mathrm{CO}_{2}$ desorption from the sorbents. By comparison with liquid PEI, the second mass loss on PEIimpregnated samples spreads over a wider range of temperatures, i.e. between 160 and 450$500^{\circ} \mathrm{C}$. Mass losses in this range of temperatures do not seem to include a significant contribution from silica de-hydroxylation because the latter is also very small for the parent SBA-15 $(<3 \%)$. Hence, the mass loss between 160 and $450-500^{\circ} \mathrm{C}$ seems to be due namely to PEI desorption/evaporation but also possibly to some surface-induced decomposition, as suggested by the asymmetry of the DTG peaks and the presence of residual carbon $(<3 \%)$ that could be oxidized by a further treatment in air at $900^{\circ} \mathrm{C}$.

Overall, it can be inferred from the different TG curves (Fig. 2) that the different sorbents are roughly stable up to $200-250{ }^{\circ} \mathrm{C}$, and in all cases they can be used confidently at least up to 160 ${ }^{\circ} \mathrm{C}$. Nevertheless, it is important to note that the decomposition of polyethyleneimine in the impregnated samples with low PEI contents starts at lower temperatures $\left(T_{\max }=287{ }^{\circ} \mathrm{C}\right.$ for 0.25PEI-SBA-15 and $283{ }^{\circ} \mathrm{C}$ for 0.25 PEI-Aerosil) than for bulk PEI $\left(\mathrm{T}_{\max }=353{ }^{\circ} \mathrm{C}\right.$ in liquid 
state) les, and shifts to higher temperatures when larger PEI amounts are impregnated $\left(\mathrm{T}_{\max }=\right.$ $319{ }^{\circ} \mathrm{C}$ for $0.75 \mathrm{PEI}-\mathrm{SBA}-15$ and $323{ }^{\circ} \mathrm{C}$ for $\left.1.6 \mathrm{PEI}-\mathrm{SBA}-15\right)$. In addition, a shoulder is also discernable at lower temperatures (around $240-250{ }^{\circ} \mathrm{C}$ ). This behavior was somewhat observed already in the literature [28]. but no clear explanation was given. As suggested by our FTIR/ATR data, the low-temperature shoulder has to be attributed to the removal of some isolated PEI oligomers confined in the mesopores whereas the contribution at higher temperatures comes from the evaporation/desorption of more densely-packed PEI molecules (PEI ad-layers on the external surface or PEI molecules in a state closer to that of the bulk liquid). This could be reasonably understood considering that PEI molecules in the bulk liquid are thermally stabilized by strong cohesive forces (such as the numerous hydrogen bonds existing between the multiple amine groups), and that these forces are less operative when PEI oligomers are dispersed in the pore system of SBA-15. Moreover, it is known that the melting point of substances decrease for nano-sized systems [29].

In addition to the mass loss related to water desorption (below $140{ }^{\circ} \mathrm{C}$ ), the TG trace of AEAPTMS-SBA-15 displays two other mass loss zones. Corresponding thermal events occur in the ranges $140-360$ and $360-700{ }^{\circ} \mathrm{C}$ and are ascribed to the two-steps decomposition of the grafted aminosilane. In that respect, it has been reported that de-hydroxylation and/or elimination of residual methoxy groups could also contribute to the mass loss observed at high temperatures [30]. Once impregnated with PEI, the organo-modified silica displayed a more pronounced mass loss between 150 and $450{ }^{\circ} \mathrm{C}\left(\mathrm{T}_{\max }=294{ }^{\circ} \mathrm{C}\right)$. It is worth noting that the "PEI DTG peak" is in that case very broad. This probably denotes some heterogeneity of surface sites and some increased interactions between the grafted aminosilane and PEI. 

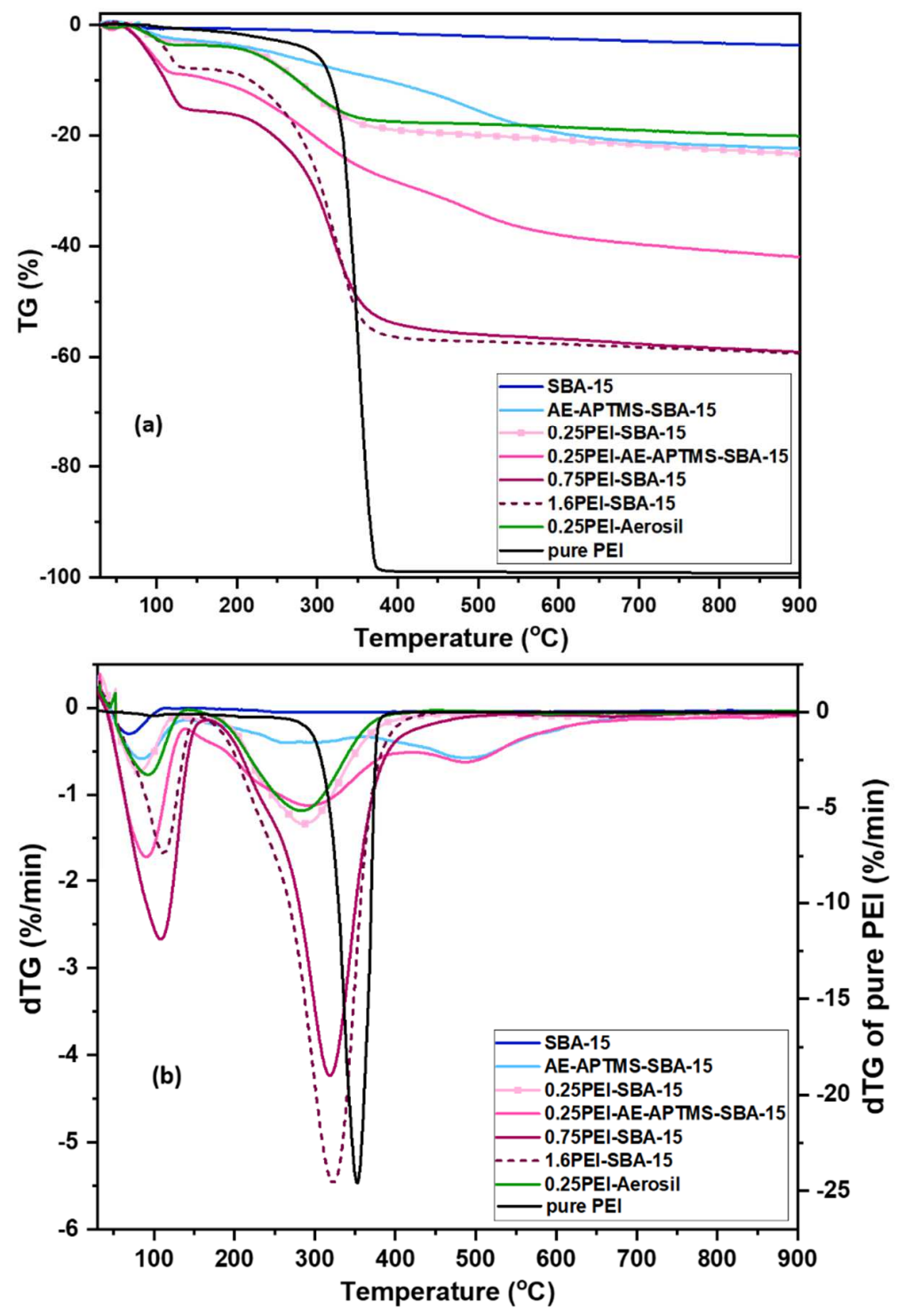

Fig. 2. (a) TG curves (wt \%) and (b) their first derivatives (DTG) for liquid PEI and PEIimpregnated sorbents during thermogravimetric experiments under $\mathrm{N}_{2}$ from 30 to $900^{\circ} \mathrm{C}(\mathrm{v}=$ $\left.10^{\circ} \mathrm{C} / \mathrm{min}\right)$

Structural and textural analyses

SAXS. Classically, the diffractogram of the parent SBA-15 (Figure S2) exhibits three peaks with relative positions $1, \sqrt{3}, 2$ and indexed to the (100), (110), and (200) reflections of the hexagonal lattice structure $(p 6 m m)$. Lattice parameter values were equal to $\mathrm{d}_{100}=9.7 \mathrm{~nm}$ and $\mathrm{a}_{0}\left(\mathrm{a}_{0}=2\right.$ $\left.\mathrm{d}_{100} / \sqrt{3}\right)=11.2 \mathrm{~nm}$. For the PEI-impregnated SBA-15 sorbents (Figure S2), it can be seen that 
the scattering intensity roughly decreases with the increase in PEI content, which is expected when mesoporous silica is heavily loaded with organics or adsorbates. The absence of shift indicates that the ordering in the porous arrangement was conserved after PEI impregnation and/or post-grafting with AE-APTMS. As expected, Aerosil and 0.25PEI-Aerosil samples displayed a flat pattern (not shown), because they do not present any ordering in their pore system.

$N_{2}$ sorptiometry at $77 \mathrm{~K}$. As expected, the parent SBA-15 material exhibits a type IV isotherm with H1 hysteresis (Fig. 3, blue) [23]. The specific surface area determined from BET method is $725 \mathrm{~m}^{2} / \mathrm{g}$. The parent material displays a rather narrow distribution of pores corresponding to an average pore diameter of $6.8 \mathrm{~nm}$ (from $\mathrm{BJH}$ model on the desorption branch) and a total pore volume of $0.96 \mathrm{~cm}^{3} / \mathrm{g}$ (mesopore volume $\left.=0.88 \mathrm{~cm}^{3} / \mathrm{g}\right)$. Table 1 .

Upon impregnation with increasing PEI contents, a rapid decrease in the textural properties can be observed (Table 1 and Fig. 3). In presence of 19 wt\% PEI (0.25PEI-SBA-15 sample), the specific surface area, the pore volume and the average pore diameter are reduced by 57, 32 and $7 \%$, respectively. Micropores present in the parent material are no more accessible (as deduced from application of $t$-plot method), and mesopores begin to be partly filled by PEI molecules. A more severe fall in textural characteristics is observed for a PEI content of $38 \mathrm{wt} \%$ (0.75PEISBA-15 sample). The capillary condensation in the mesopores is shifted to lower $\mathrm{P} / \mathrm{P}_{0}$ values and the steepness of the hysteresis decreases (Fig. 3, violet). The new hysteresis can be identified as H4 type, representing narrow slit-like pores with irregular shapes and broad pore size distribution [31]. As expected, the pore size distribution for 0.75PEI-SBA-15 is no more uniform (bimodal pore size distribution at 6.3 and $4 \mathrm{~nm}$ ) and a drastic loss in specific surface area (- 94\%) and pore volume (- 85\%) is observed (Table 1). The bimodal size distribution is indicative of necking, due 
to the presence of a PEI oligomer at the entrance or further in the mesopores. For 1.6PEI-SBA15 (51 wt\% PEI), the flat isotherm (Fig. 3, brown) and the absence of specific surface area indicate that the pores are no more available for the $\mathrm{N}_{2}$ probe. In other words, although the hexagonal mesoporous structure is still preserved, the access to the porosity is now totally plugged by PEI. Hence, the presence of multiple PEI ad-layers or large PEI domains on the external surface is expected. Such domains also probably exist at lower PEI contents but to a lesser extent.

Rather similar phenomena are observed for the organo-modified (post-grafted) silica (Fig. 3, pink). Classically, the SBA-15 sample previously grafted with aminosilane (AE-APTMS-SBA15) presents a significant loss of specific surface area and a moderate decrease in pore size ($15 \%$ ) due to the random anchoring of organic species along the pore walls. By impregnation of PEI (11 wt\%), a bimodal pore size distribution is also observed (5.8 and $3.7 \mathrm{~nm})$, which can be linked to a further restriction of the size of some mesopores by interacting PEI oligomers. Overall, the behavior of the amino-functionalized SBA-15 follows a trend rather similar to that of the parent material, provided that the total $\mathrm{N}$ content is considered, and not simply the PEI content.

The fumed silica (Aerosil 200) displays an isotherm shape (Figure S3) typical of non-porous or macroporous material (type II), due to an unrestricted monolayer-multilayer adsorption. However, the hysteresis reflects the presence of an external mesoporosity between the silica nanoparticles (10-20 $\mathrm{nm}$ in size). The impregnation of 20\% PEI decreases the specific surface area of Aerosil from 177 to $85 \mathrm{~m}^{2} / \mathrm{g}$ (corresponding to a $52 \%$ loss), and has also an effect on the pore size distribution in the mesoporous range (Figure S3). For this type of nanosilica, it could be 
concluded that the PEI molecules are preferentially settled in the voids of size 2-15 nm located between the silica nanoparticles.
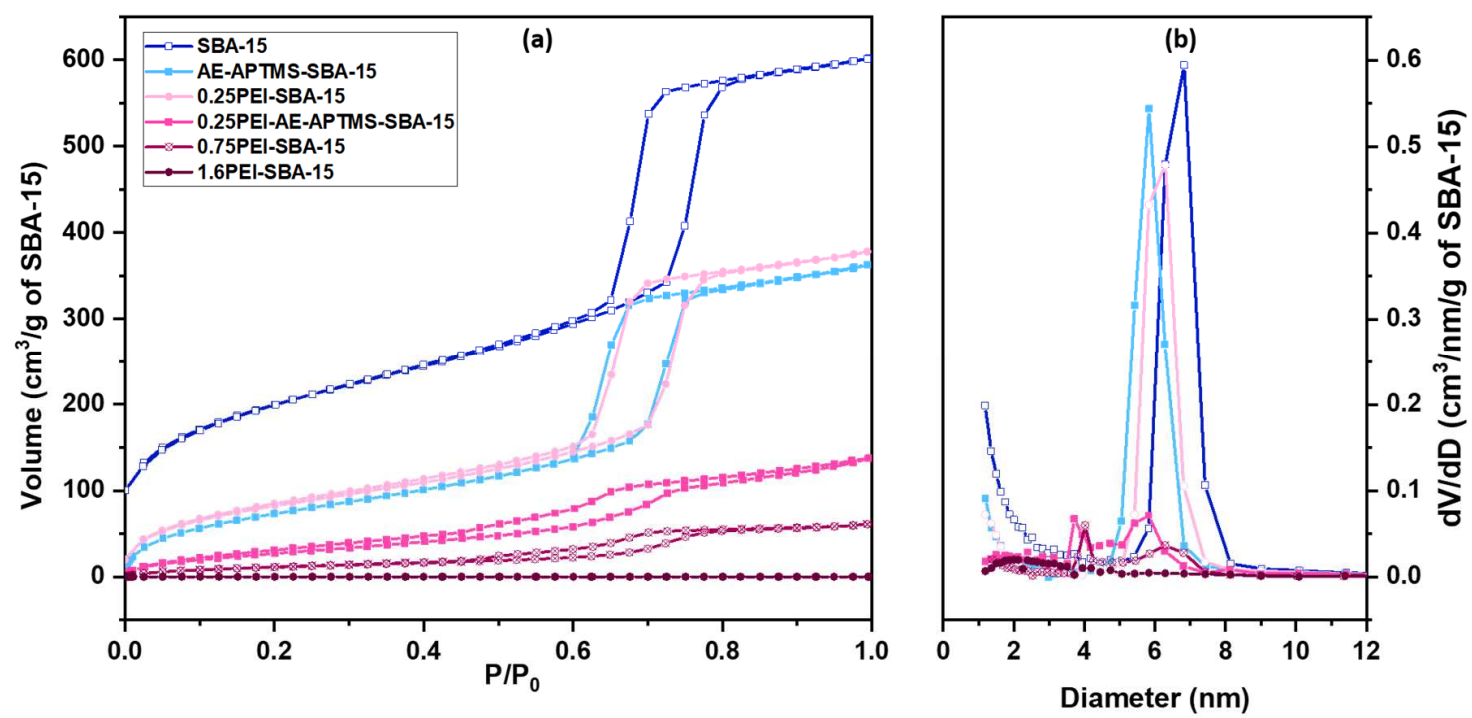

Fig. 3. Textural properties of PEI-impregnated SBA-15 sorbents. (a) $\mathrm{N}_{2}$ adsorption-desorption isotherms at $77 \mathrm{~K}$; (b) pore size distributions determined from $\mathrm{BJH}$ method on the desorption branch

\subsection{I2 adsorption on PEI-impregnated sorbents}

Liquid-phase tests at $20^{\circ} \mathrm{C}$. Iodine adsorption capacities ( $\mathrm{QI}_{2}$ in $\mathrm{mg} / \mathrm{g}$ ) deduced from UV-Visible spectrophotometric measurements at $\lambda_{\max }=523 \mathrm{~nm}$ (see experimental part for details) are reported in Table 2. Without any modification of their surface properties, the parent SBA-15 and Aerosil materials have a very limited ability to adsorb iodine $\left(\mathrm{QI}_{2}=87\right.$ and $72 \mathrm{mg} / \mathrm{g}$, respectively). As the PEI content in SBA-15 increased to $19 \mathrm{wt} \%$ and then to $38 \mathrm{wt} \%$, the $\mathrm{I}_{2}$ adsorption capacity passes to $1090 \mathrm{mg} / \mathrm{g}$ and then almost doubles to $2060 \mathrm{mg} / \mathrm{g}$. Overall, a general correlation was obtained by plotting the $\mathrm{QI}_{2}$ values in function of the $\mathrm{N}$ content (wt $\%$ ) of the sorbents (Fig. 4). The latter shows an almost linear increase up to a $\mathrm{N}$ content of $12-13 \mathrm{wt} \%$. However, for the highest PEI content $(51 \mathrm{wt} \%, \% \mathrm{~N}=16.6)$, a moderate decrease is observed (1982 mg/g), which could be explained by our characterization data. It was shown that the 
specific surface area and pore volume of PEI-SBA-15 quickly decreased as PEI molecules progressively filled the pores. For the 1.6PEI-SBA-15 material, our adsorption results indicate that some $\mathrm{I}_{2}$ molecules do not have access to a significant part of the adsorption sites located in the mesopores because of their entrance or internal space are totally plugged by PEI molecules. In that case, it could be expected that $\mathrm{I}_{2}$ adsorption involves the amine groups located in PEI $a d$ layers on the external surface and PEI nests at the pore mouth rather than those located in the internal porosity. This hypothesis seems to be comforted by the aspect of the samples at the end of the test: while the 0.25 and 0.75PEI-SBA-15 samples still preserved their powdered aspect, the 1.6PEI-SBA-15 changed from sticky (before test) to almost stone-like after test, indicating the occurrence of strong interactions between PEI and $I_{2}$ on the external surface. Overall, our results seem to show that iodine adsorption takes place mostly in the mesopores for low-medium PEI contents and outside of the porosity or at the pore mouth when the pores are getting totally filled with PEI. Consistently with our results, literature studies also indicate optimum organic loadings in the range 30-50 wt $\%$ in the case of $\mathrm{CO}_{2}$ adsorption by PEI-silica materials $[24,32-$ 34]. At higher PEI contents, the diffusion of $\mathrm{CO}_{2}$ was found to be severely hindered by the high concentration of PEI molecules in the porosity. Indeed, a parallel situation is found in the present study for iodine adsorption.

Table 2. Iodine adsorption capacities (mg/g) determined from liquid and gas-phase adsorption tests

\begin{tabular}{|c|c|c|c|c|}
\hline \multirow[t]{2}{*}{ Adsorbent } & \multicolumn{2}{|c|}{$\begin{array}{l}\text { Liquid phase retention tests } \\
\qquad\left(20^{\circ} \mathrm{C}\right)\end{array}$} & \multicolumn{2}{|c|}{$\begin{array}{c}\text { Gas-phase retention tests } \\
\left(60^{\circ} \mathrm{C}\right)\end{array}$} \\
\hline & $\mathrm{QI}_{2}(\mathrm{mg} / \mathrm{g})$ & $\mathrm{I}_{2} / \mathrm{N}$ & $\mathrm{QI}_{2}(\mathrm{mg} / \mathrm{g})$ & $\mathrm{I}_{2} / \mathrm{N}$ \\
\hline SBA-15 & 87 & - & 17.6 & - \\
\hline 0.25PEI-SBA-15 & 1090 & 0.97 & 953 & 0.86 \\
\hline 0.75PEI-SBA-15 & 2062 & 0.93 & 2106 & 0.94 \\
\hline 1.6PEI-SBA-15 & 1982 & 0.67 & 1916 & 0.64 \\
\hline AE-APTMS-SBA-15 & 809 & 0.8 & 657 & 0.64 \\
\hline 0.25PEI-AE-APTMS-SBA-15 & 1419 & 0.84 & 1185 & 0.7 \\
\hline Aerosil & 72 & - & 0 & - \\
\hline
\end{tabular}


The effect of organic functionalization by an aminosilane containing two $\mathrm{N}$ atoms (AE-APTMS) was also studied on iodine adsorption. In the presence of AE-APTMS only, the adsorption capacity of SBA-15 jumped from 87 to $809 \mathrm{mg} / \mathrm{g}$. Although AE-APTMS-SBA-15 (\%N= 5.6) is slightly under the trend observed for PEI-containing sorbents, it shows that the most important parameter for $\mathrm{I}_{2}$ adsorption is the presence of accessible amine groups. By impregnating the postgrafted SBA-15 material with $11 \mathrm{wt} \%$ PEI $\left(\% \mathrm{~N}=3.7\right.$ corresponding to $\left.\% \mathrm{~N}_{\text {total }}=9.3\right)$, the adsorption capacity further increased to $1419 \mathrm{mg} / \mathrm{g}$. The increase in adsorption capacity $(+610$ $\mathrm{mg} / \mathrm{g}$ ) is similar to what could be expected in the absence of AE-APTMS, indicating that the presence of AE-APTMS on the pore walls did neither lead to an appreciable increase nor a decrease in the adsorption performance of PEI (at the content studied). A different behavior could be expected for instance if the presence of organosilane would significantly promote a more uniform dispersion of PEI in the pores, or on the contrary, restrict its access to some parts of the pores by steric hindrance.

The effect of the porosity can be further addressed by comparing the performances of SBA-15 and Aerosil materials with similar PEI contents (19-20 wt\%). For Aerosil, the iodine adsorption performance was enhanced from 72 to $1156 \mathrm{mg} / \mathrm{g}$ after impregnation with PEI $(\% \mathrm{~N}=6.4)$. This adsorption capacity is very similar to the one found for 0.25 PEI-SBA-15 ( $\% \mathrm{~N}=6.1$ ). It was reported in section 03.1 that PEI molecules impregnated on Aerosil material are located namely in the mesoporous voids between silica nanoparticles. Hence, this shows that the size and type of pores is not the most influent parameter for $\mathrm{I}_{2}$ adsorption, the retention being driven by chemical interactions between the sorbents and iodine. 


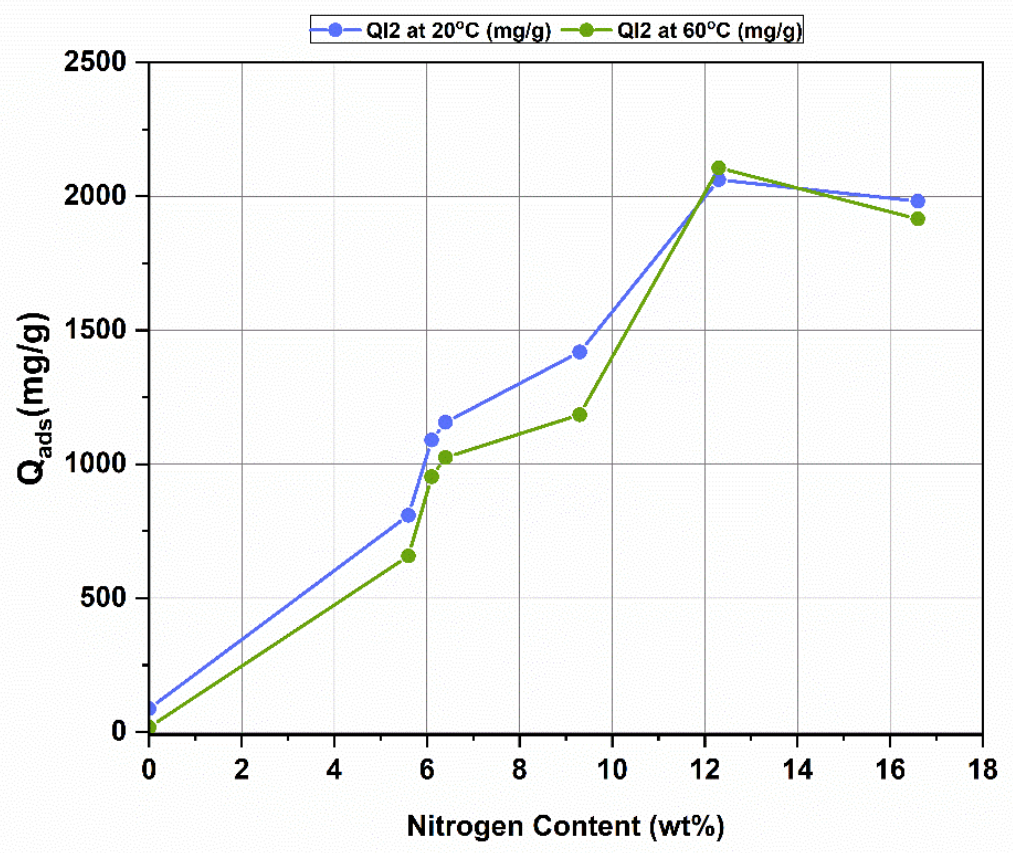

Fig. 4. Liquid and gas-phase $\mathrm{I}_{2}$ adsorption capacities $(\mathrm{mg} / \mathrm{g})$ plotted in function of the $\mathrm{N}$ content (wt $\%)$ of the impregnated samples

Gas-phase tests. The results of static gas-phase adsorption experiments performed at $60{ }^{\circ} \mathrm{C}$ during $12 \mathrm{~h}$ (under iodine partial pressure of $356+/-20 \mathrm{~Pa}$ ) are described now. When plotted against the $\mathrm{N}$ content of the sorbents, the adsorption capacities calculated gravimetrically from those experiments (green line on Fig. 4) show a rather identical evolution to those measured in liquid phase at $20{ }^{\circ} \mathrm{C}$ (blue line on Fig. 4), but the values were in most cases slightly lowered by $3-18 \%$. Nevertheless, it is important to mention that in the sets of gas-phase adsorption data obtained at $60^{\circ} \mathrm{C}$ and presented in Table 2 and Fig. 4, the contribution of weakly-bonded species (physisorbed species which could be easily removed by a displacement of equilibrium) was eliminated by a $7 \mathrm{~h}$ evacuation treatment. During this period, it was checked that the adsorbents lost about 35-40 wt $\%$ of their overall adsorption capacity. Nevertheless, in nuclear context, it is more important to consider the stable iodine adsorption capacity, i.e. the fraction which cannot 
be removed by a simple change of conditions. Hence, the adsorption capacities reported in Table 2 and Fig. 4 should be regarded as mostly site-specific and the corresponding samples were used thereafter for ex situ characterization studies (part 3.3). Overall, this indicates that iodine chemisorption (or strong adsorption) prevails in both liquid and gas-phase media and at different temperatures.

More information on the "nitrogen use" by the sorbents for iodine trapping can be deduced from calculations of atomic $\mathrm{I}_{2} / \mathrm{N}$ ratio. Remarkably, these ratios were found close to 1 (or slightly below) for the sorbents when the PEI content is below $\approx 40 \mathrm{wt} \%$. This indicates that one $\mathrm{I}_{2}$ molecule can be adsorbed in average in the vicinity of a $\mathrm{N}$ atom and therefore, a rather optimal use of amine functionalities. Nevertheless, for PEI content above $50 \mathrm{wt} \%$, the $\mathrm{I}_{2} / \mathrm{N}$ ratio decreases to values close to 0.65 due to limited accessibility. This behavior is somewhat close to that reported in the study of Chen et al. [32] in the case of $\mathrm{CO}_{2}$ adsorption on amino-modified resins. However, the $\mathrm{I}_{2} / \mathrm{N}$ ratio computed in this study is about three times higher than their $\mathrm{CO}_{2} / \mathrm{N}$ ratio, indicating that the amino groups have a much higher affinity for iodine than for $\mathrm{CO}_{2}$.

In the present study, the $\mathrm{I}_{2} / \mathrm{N}$ ratio was found to be a bit degraded in presence of AE-APTMS or when this aminosilane is introduced before PEI impregnation. Without certainty, this could be due to some differences existing in density or types of amine groups present (primary, secondary and tertiary amines exist in ratio 1:2:1 in branched PEI whereas the ratio primary/secondary is 1:1 for AE-APTMS) but it is also probable that the packing and accessibility of PEI in the pores could be also simply affected by the presence of the aminosilane.

Finally, on Fig. 5 is displayed the breakthrough curve obtained for the best sorbent (0.75PEISBA-15 with 38 wt\% PEI) under dynamic testing conditions. It was obtained when the sorbent 
was continuously exposed to flowing iodine $\left(\left[\mathrm{I}_{2}\right]_{0}=1240 \mathrm{ppm} / \mathrm{balance}\right.$ with $\operatorname{Ar}(100 \mathrm{~mL} / \mathrm{min})$ at a higher temperature $\left(100{ }^{\circ} \mathrm{C}\right.$, using on-line UV-Vis detection) under conditions closer to what is expected for FCVS application. On Fig. 5, it can be seen that all the incoming iodine is adsorbed by the sorbent during almost $5 \mathrm{~h}$, this period corresponding to total retention. After breakthrough, the iodine concentration in the gas phase slowly increased up to the nominal concentration, indicating a saturation of the sorbent bed after $7 \mathrm{~h}$. From the quantitative exploitation of this curve (Fig. 5), it can be deduced that the adsorption capacities at breakthrough and at saturation are equal to $Q_{\text {breakthrough }}=1919 \mathrm{mg} / \mathrm{g}$ and $\mathrm{Q}_{\text {sat }}=2127 \mathrm{mg} / \mathrm{g}$, respectively. The latter is comparable to the results obtained under gas-phase conditions at $60{ }^{\circ} \mathrm{C}(2106 \mathrm{mg} / \mathrm{g})$ and in liquid-phase at 20 ${ }^{\circ} \mathrm{C}(2062 \mathrm{mg} / \mathrm{g})$. Hence, it seems obvious that PEI-SBA-15 sorbents are capable to trap high amounts of iodine, up to $2 \mathrm{~g} / \mathrm{g}$ and above, in the range $20-100{ }^{\circ} \mathrm{C}$. In the literature, the effect of temperature on PEI-silica sorbents was examined in the case of $\mathrm{CO}_{2}$ adsorption, and adverse effects were reported to take place[28,33,37,40]: (i) a decrease in the adsorption capacity with temperature due to the exothermicity of the reaction between $\mathrm{CO}_{2}$ and amines (forming ammonium carbamates); (ii) an improved mobility of PEI molecules with the temperature, this promoting the diffusion of the adsorbate towards the adsorption sites with limited accessibility (deep in the pores), therefore enhancing the adsorption capacity. In the present study, it is possible that a temperature of $100{ }^{\circ} \mathrm{C}$ could partly overcome the impact of pore blockage at high PEI loadings, but it will also decrease the occurrence of physisorption processes, overall resulting in only small variations of the iodine adsorption capacity over the range $20-100{ }^{\circ} \mathrm{C}$.

Finally, it is worth noting that the adsorption capacities of $\approx 2 \mathrm{~g} / \mathrm{g}$ found at PEI contents in the range 38-51 wt $\%$ are at least comparable or even higher than those found in the literature for the best functionalized MOFs [10], COFs [12] and porous polymers (POPs) [13]. They are also 4-5 
times greater than those of the best silver zeolites (silver faujasites with 23-35 wt\% silver), which are some of the sorbents currently studied for potential application in nuclear power plants $[7,8]$.

Some insights onto the thermal stability of adsorbed species and the mechanisms of iodine adsorption in presence of PEI will be given in the next sections.

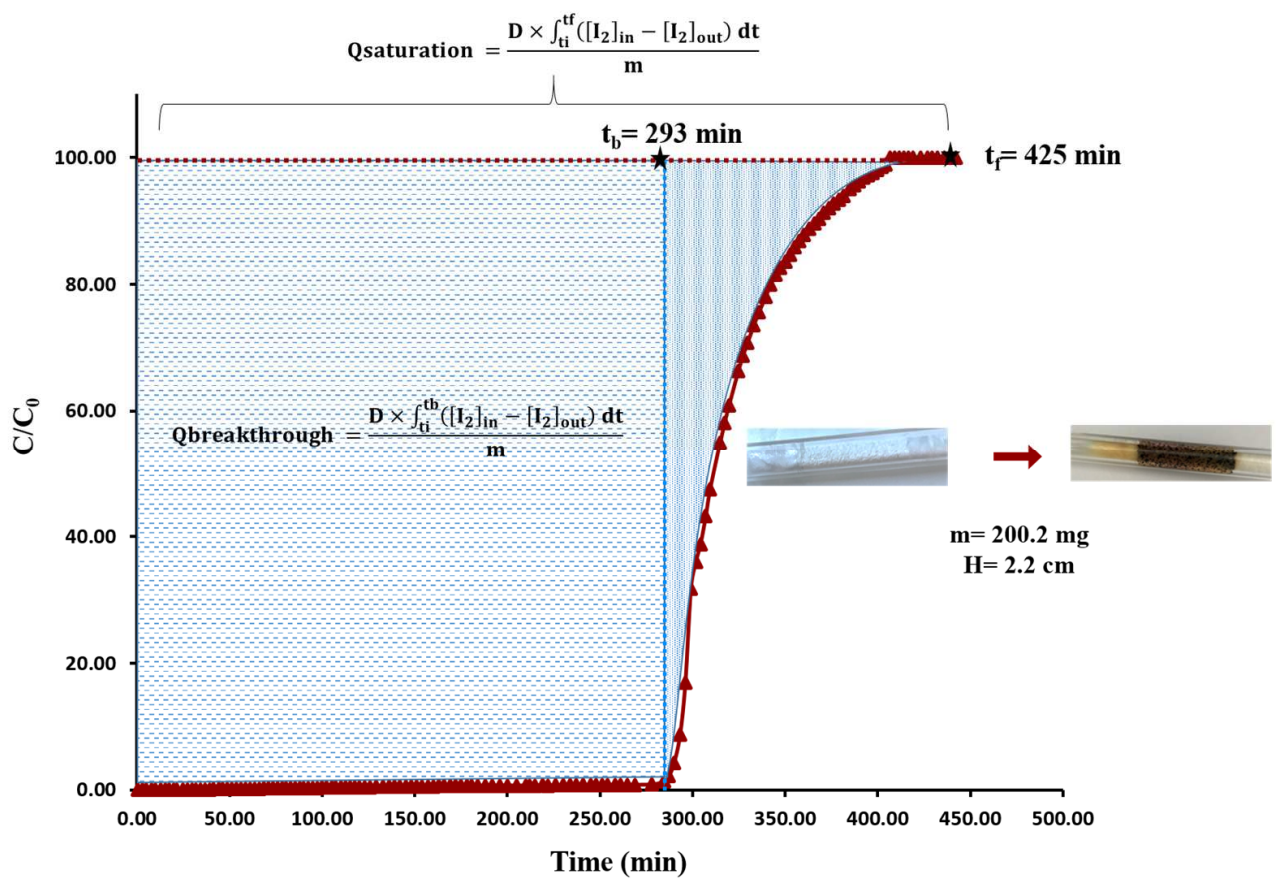

Fig. 5. Breakthrough curve obtained at $100^{\circ} \mathrm{C}$ for the $0.75 \mathrm{PEI}-\mathrm{SBA}-15$ sorbent during a dynamic gas-phase iodine adsorption test, $\left[\mathrm{I}_{2}\right]_{0}=1240 \mathrm{ppm} / \mathrm{Ar}(100 \mathrm{~mL} / \mathrm{min})$

\subsection{After-test characterization}

Depending on the adsorption capacity, the color of the samples changed from orange-brown to almost black (see for instance inlet on Fig. 5) at very high iodine uptake. These samples were submitted to different characterization procedures in order to have more information on the nature of the interactions existing between adsorbed iodine species and the sorbents as well as the thermal stability of iodine $a d$-species. 
Thermogravimetric analyses of iodine desorption. Once the static adsorption tests at $60{ }^{\circ} \mathrm{C}$ were completed (see previous part), the iodine-loaded samples were analyzed by TGA (Fig. 6) under the same conditions used in part $0\left(\mathrm{~N}_{2}\right.$ flow, $\mathrm{v}=10{ }^{\circ} \mathrm{C} / \mathrm{min}$ from 30 to $\left.900{ }^{\circ} \mathrm{C}\right)$. Overall, a rather good matter balance was found between the adsorption capacities measured in liquid and gasphase tests (Table 2) and the amounts of iodine desorbed by TGA (if both expressed in $\mathrm{mgI}_{2} / \mathrm{g}_{\text {sorbent }}$ or in $\%$ of the initial mass), indicating the absence of significant iodine incorporation into the silicic framework.

By comparison with Fig. 2, it can be observed that the first mass loss occurring below $c a 100{ }^{\circ} \mathrm{C}$ and previously assigned to adsorbed moisture and/or $\mathrm{CO}_{2}$ is no more observed for all the samples (Fig. 6), indicating a preference of the sorbents for iodine. The two main mass losses observed in the range $150-450{ }^{\circ} \mathrm{C}$ are both related to iodine desorption and PEI evaporation/decomposition (Fig. 6). The first zone between 120 and $230{ }^{\circ} \mathrm{C}$ is almost exclusively assigned to desorption of adsorbed iodine because the $\mathrm{T}_{\max }$ values $\left(180-200{ }^{\circ} \mathrm{C}\right)$ match rather well with the boiling point of iodine $\left(184{ }^{\circ} \mathrm{C}\right)$ and it was also reported that the rate of PEI evaporation/decomposition is slow at these temperatures (part 0) This form of adsorbed iodine will be referred thereafter as $\left(\mathrm{I}_{2}\right)_{\mathrm{n}}$ and it is rather similar to iodine pellets.

The second peak $\left(\mathrm{T}_{\max }=300-320{ }^{\circ} \mathrm{C}\right)$ on DTG curves $($ Fig. 6(b)) is sharper and also more intense than those found for the as-synthesized samples (Fig. 2(b)). It should probably be correlated with the decomposition of some ionic forms of iodine $\left(\mathrm{I}_{\mathrm{n}}{ }^{-}, \mathrm{I}^{+} ..\right)$, which should be more thermally stable than $\left(\mathrm{I}_{2}\right)_{\mathrm{n}}$ solid species due to increased interactions with some positivelycharged amino groups in PEI. In the upper range of temperatures, the desorption of iodine between 350 and $450{ }^{\circ} \mathrm{C}$ (as observed as a shoulder of the main desorption peak at $300-320^{\circ} \mathrm{C}$ ) could arise from iodine forms particularly difficult to remove, such as species deeply buried in 
the pores or existing as hard precipitate with PEI. In that respect, the 1.6PEI-SBA-15 sample had a stone-like aspect after test, different from the others and displayed a supplementary peak at ca $700^{\circ} \mathrm{C}($ Fig. 6(b)).
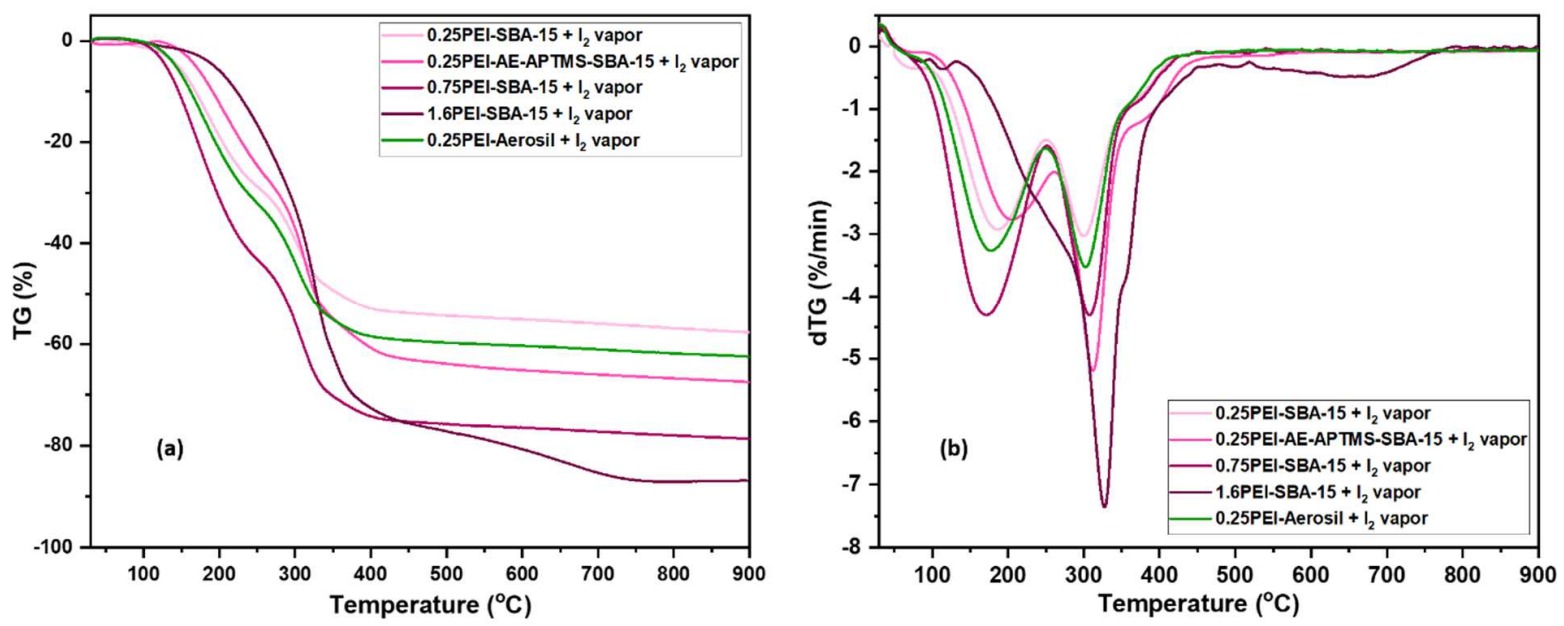

Fig. 6. TG (a) and DTG (b) curves corresponding to the desorption of adsorbed iodine from PEIimpregnated sorbents. Conditions: $\mathrm{N}_{2}$ atmosphere, heating from 30 to $900^{\circ} \mathrm{C}\left(\mathrm{v}=10^{\circ} \mathrm{C} / \mathrm{min}\right)$

Spectroscopic analyses on the spent sorbents

DRS-UV-Vis spectroscopy. The color evolution of the sorbents before-after iodine adsorption tests was confirmed by DR-UV-Vis spectroscopy (Fig. 8). First of all, it was found that this technique is highly sensitive to the presence of very low amounts of adsorbed iodine, as witnessed from the presence of bands at 290 and $370 \mathrm{~nm}$ in the spectra of the parent SBA-15 and Aerosil sorbents (with very pale yellow color and low adsorption capacities (Table 2). These bands are assigned to a charge-transfer complex, formed through weak interactions between iodine and the lone pair of oxygen atoms at the silica surface. These bands share similar characteristics to $\mathrm{I}_{3}{ }^{-}$and/or $\mathrm{I}_{5}^{-}$ions that absorb in the same spectral region between 290 and 360 nm [37]. 
For the spent PEI-impregnated sorbents, their dilution was essential (with the non-absorbing SBA-15 powder as dilutant) in order to get DR spectra free of artefacts caused by the spectral saturations. Before being contacted with iodine, the PEI-impregnated samples were characterized by a rather strong absorption in the $200-250 \mathrm{~nm}$ range due to $\mathrm{n} \rightarrow \sigma^{*}$ transitions of amino groups in polyethyleneimine (Figure S 4). After exposure to iodine vapor at $60^{\circ} \mathrm{C}$, new bands at 280 300 and 360-380 $\mathrm{nm}$ were observed for all the spent samples. Remarkably, these bands were found to be rather similar to those found during preliminary experiments in homogeneous cyclohexane solution (bands at 290 and $380 \mathrm{~nm}$ ), when unsupported PEI was directly contacted with iodine. During these experiments, the pink-violet color of iodine-cyclohexane solution (corresponding to an absorption band at $523 \mathrm{~nm}$ in the visible region) disappeared and turned to yellow-brownish (corresponding to two bands at $c a 290$ and $380 \mathrm{~nm}$ ) as PEI was progressively added (Figure S 5). Moreover, these complexes were found to rapidly settle as solid precipitates at the bottom and on the walls of the vessel due to their limited solubility and/or to an increase in molecular weight due to iodine uptake (flocculation-like process between positively-charged amines and negative iodine species). At the surface of PEI-impregnated sorbents, it is postulated that charge transfer complexes are formed in an initial step through halogen bonding between amino species and iodine, followed by total charge dissociation with evolution to $\mathrm{I}_{\mathrm{n}}{ }^{-}, \mathrm{NH}_{\mathrm{x}}{ }^{+}$ species (the latter being responsible of the observed absorptions).

On the other hand, another band can be identified in the UV region around $230 \mathrm{~nm}$, accompanied with a broad absorption in the visible region (near 500-520 nm). Owing to some similarities existing with the spectra of $\mathrm{I}_{2}$-cyclohexane solutions in absence of PEI, this set of bands correspond to a molecular form of adsorbed iodine $\left(\mathrm{I}_{2}\right)_{\mathrm{n}}$, also evidenced in TGA measurements. 
Hence, it was confirmed that both ionic and neutral adsorbed species co-exist at the surface of the sorbents.

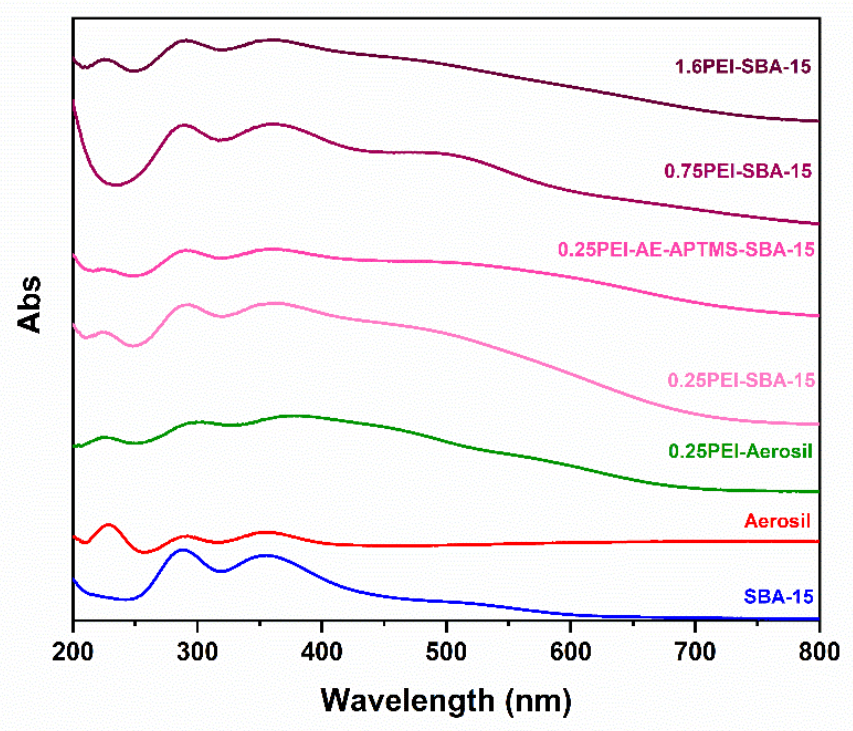

Fig. 7. DR-UV-Vis spectra of the spent PEI-impregnated sorbents (after iodine treatment at $\left.60^{\circ} \mathrm{C}\right)$

Vibrational spectroscopies. In contrast with FTIR, vibrations of diatomic homonuclear molecules, such as $\mathrm{I}_{2}$, can be directly observed by Raman spectroscopy [38]. For instance, solid iodine has a Raman-active mode at $180 \mathrm{~cm}^{-1}$, whereas molten and gaseous iodine are characterized by bands at 194 and $213 \mathrm{~cm}^{-1}$, respectively [39,40]. Moreover, the frequency of the I-I symmetric stretching vibration (i.e. the length of the bond) does not vary only according to the iodine physical state but also by forming adducts with a donor (D), such as in I-I $\cdots$ D chargetransfer complexes. For the PEI-impregnated sorbents treated with $\mathrm{I}_{2}$ vapor (Fig. 8), a broad absorption is observed at frequencies below those of solid $\left(\mathrm{I}_{2}\right)_{\mathrm{n}}$ species. Such lowering in bond order is the consequence of the coordination of $\mathrm{I}_{2}$ by amino species and the decrease of the force constant thanks to electronic density donation into the $\sigma^{*}$ antibonding orbital of the halogen 
[41,42]. For the different PEI-SBA-15 sorbents (Fig. 8(A)), the Raman spectra display more or less resolved maxima at $147,156,168 \mathrm{~cm}^{-1}$. According to the extensive study on the Raman signature of polyiodides and donor- $\mathrm{I}_{2}$ adducts by Deplano et al. [43], coordinated $\mathrm{I}_{\mathrm{n}}{ }^{\mathrm{x}-}$ species of different chain lengths or charge are responsible of the three first peaks. In fact, these $\mathrm{I}_{\mathrm{n}}{ }^{\mathrm{x}-}$ species are not real entities but must be better viewed at linear or bent adducts formed between $\mathrm{I}^{-}$or $\mathrm{I}_{3}^{-}$ and $\mathrm{I}_{2}$ through donor-acceptor interactions. Besides the above-mentioned peaks, another contribution at $180 \mathrm{~cm}^{-1}$ (existing as a shoulder on Fig. 8(A)) can also be identified especially for the sample having the higher adsorption capacity (0.75PEI-SBA-15). Even if this band was also assigned in the literature to some $\mathrm{I}_{2}$ moieties in coordinated polyiodides, it could here also indicate the presence of solid (physisorbed) iodine (see Fig. 8(B)), in good agreement with TGA and DRS-UV-Vis data. For PEI-impregnated Aerosil, rather similar features were also observed. An increase in the laser power during spectral recording was found to enhance the intensity of the peaks at 182 and $192 \mathrm{~cm}^{-1}$ in contrast with the other contributions at lower frequencies (Fig. 8(B)). This has to be interpreted by the decomposition of unstable $\mathrm{I}_{\mathrm{n}}^{-}$species by the heat generated by the laser and the re-deposition of generated iodine vapor as solid $\mathrm{I}_{2}$. Hence, care has to be taken when recording the spectra of those adsorbed polyiodides. 

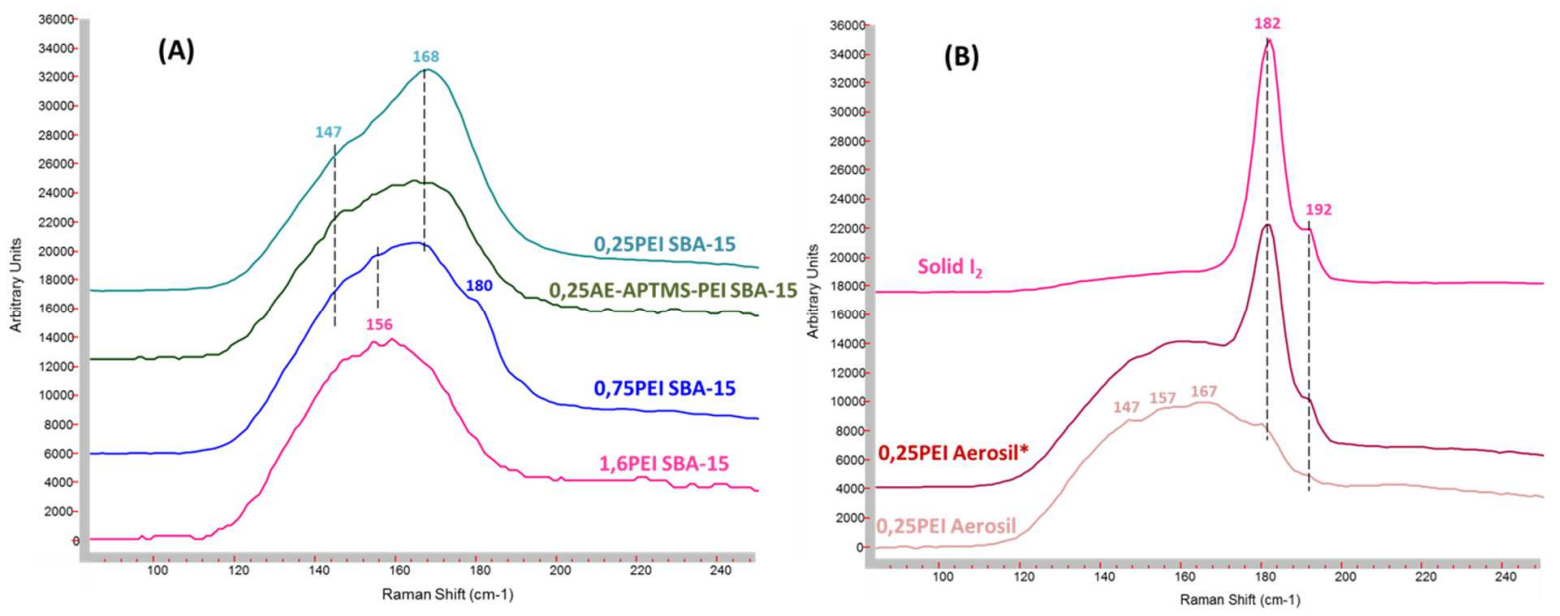

Fig. 8. Raman spectra of the spent sorbents after iodine adsorption. PEI-SBA-15 sorbents (A), and PEI-Aerosil (B). The spectrum marked with a star corresponds to an increase of the laser power from 40 to $100 \%$.

This Raman spectroscopic study was also completed by FTIR/ATR analyses of the sorbents after iodine adsorption tests (Fig. 9). Since all iodine-related vibrations (I-I and N-I) could only be detected in the far IR below $400 \mathrm{~cm}^{-1}$, only the perturbations induced on the spectrum of PEI could be assessed in the mid-IR range. By comparison with the FTIR/ATR spectra of the PEIimpregnated materials not exposed to iodine (Fig. 1), rather important changes were noticed for the $\mathrm{I}_{2}$-loaded samples resulting in a significant distortion of the baseline, band broadening, with alteration of peak intensities, especially for sorbents with high PEI contents (Fig. 9). In order to gain more confidence on the assignment of those spectral features, a comparison was made with the spectra of the brownish precipitates formed from liquid-phase reaction of PEI with iodine solutions (in cyclohexane) in the absence of SBA-15. The most prominent changes were associated with some shifts or changes in the extinction coefficient of all amine-related vibrations due to the interactions formed between $I_{2}$ and the primary, secondary and tertiary amines of PEI (see Figure S7). Characteristic bands due to the formation of N-iodine complexes 
are now identified in the precipitates at $c a 3270\left(\mathrm{NH}_{\mathrm{x}}\right.$ stretch), 2930, 2825 (stretches of ethylene moieties), 1650, 1600-1560 $\left(\mathrm{NH}_{\mathrm{x}}\right.$ bend), $1470-1450\left(\mathrm{CH}_{2}\right.$ bend), in the $1300-1000 \mathrm{~cm}^{-1}$ region (C-N stretch), and around 900-750 $\mathrm{cm}^{-1}\left(\mathrm{NH}_{\mathrm{x}}\right.$ wag) [26]. Except the $\mathrm{NH}_{\mathrm{x}}$ wag, most of other amine-related bands gained stronger intensities after contact with iodine solutions. Upon the addition of few drops of water, an immediate enhancement of the bands at 1559, 1466 and 1302 $\mathrm{cm}^{-1}$ was observed as well as the build-up of an absorption continuum spreading between 3600 and $2000 \mathrm{~cm}^{-1}$. According to literature studies [38,44,45], many $\mathrm{D} \cdot \mathrm{I}_{2}$ complexes with amines as donors are rather stable in inert mediums, but can form ionic compounds (due to complete charge transfer) when present in an ionizing medium (such as water, even if present as traces). Hence, the continuum is ascribed to the overlapping between the stretching vibrations of charged and neutral primary and secondary amines and those of ethylene groups, as well as Fermi-resonance enhanced combination-bands of amine salts [26]. In presence of iodine and water, the intrinsic bandwidths are expected to be further broadened through halogen bonding (with iodine) or hydrogen bonding (with water).

For the PEI-impregnated sorbents exposed to $I_{2}$ vapors (Fig. 9), rather similar features were obtained (except those associated with silica framework vibrations). For the highest iodine uptakes (0.75PEI-SBA-15 and 1.6PEI-SBA-15, most of the bands related to $\mathrm{NH}_{\mathrm{x}}$ of $\mathrm{CH}_{2}$ stretches were considerably broadened and overlapped, giving rise again to a broad absorption continuum between 3600 and $1800 \mathrm{~cm}^{-1}$. Hence, it seems reasonable to ascribe the perturbations observed in PEI frequencies to the co-existence of both neutral and ionic iodine species. On the other hand, the increase of the shoulder at $935 \mathrm{~cm}^{-1}$ (linked to the broad absorption ca $3455 \mathrm{~cm}^{-1}$ ) has probably to be ascribed to the formation of silanol species. Without certainty, this could 
possibly indicate a progressive amorphization of SBA-15 mesostructured network when the iodine uptake reaches a certain level.

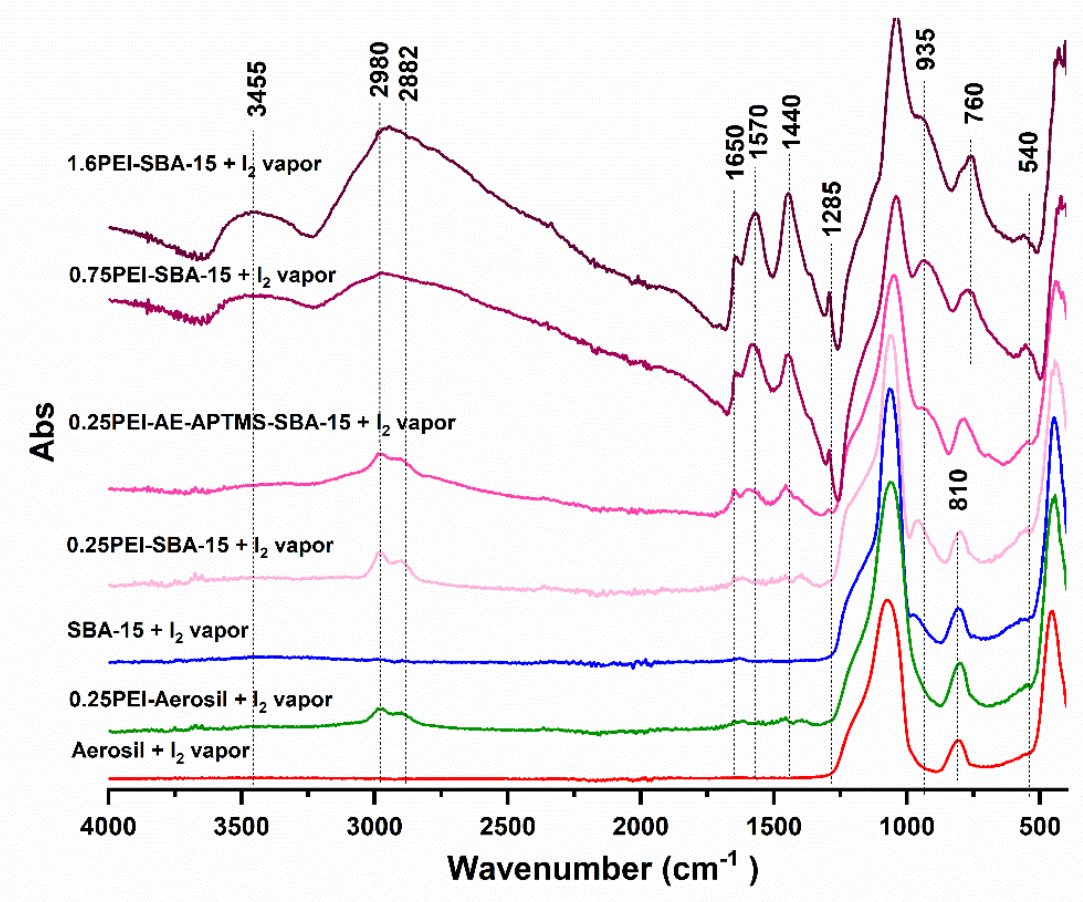

Fig. 9. FTIR/ATR spectra of PEI-impregnated samples after the adsorption of iodine vapor

Overall, the comparison of the data obtained from the different spectroscopic characterizations done on the spent sorbents (DR-UV-Vis, IR, Raman) with those obtained from TGA desorption experiments indicates that both ionic $\left(\mathrm{D}^{+} / \mathrm{I}_{\mathrm{n}}{ }^{-}\right)$, complexed $\left(\mathrm{D}^{-} \mathrm{I}_{2}\right)$ and molecular forms (such as physisorbed or solid iodine) of adsorbed iodine co-exist on the sorbents after $\mathrm{I}_{2}$ exposure. Scheme 1 represents schematically the processes on-going during iodine adsorption of PEIimpregnated silicas. Polyiodide entities $\left(\mathrm{I}_{\mathrm{n}}^{-}\right)$that do not exist as discrete entities, but as a combination of $\mathrm{I}_{2}$ molecules and the Lewis base $\mathrm{I}^{-}$and/or $\mathrm{I}_{3}{ }^{-}$and their existence can be promoted by the presence of co-adsorbed water or silanol groups [46]. 
Scheme 1. The proposed mechanism for the interaction between the adsorbed iodine and the impregnated PEI entities

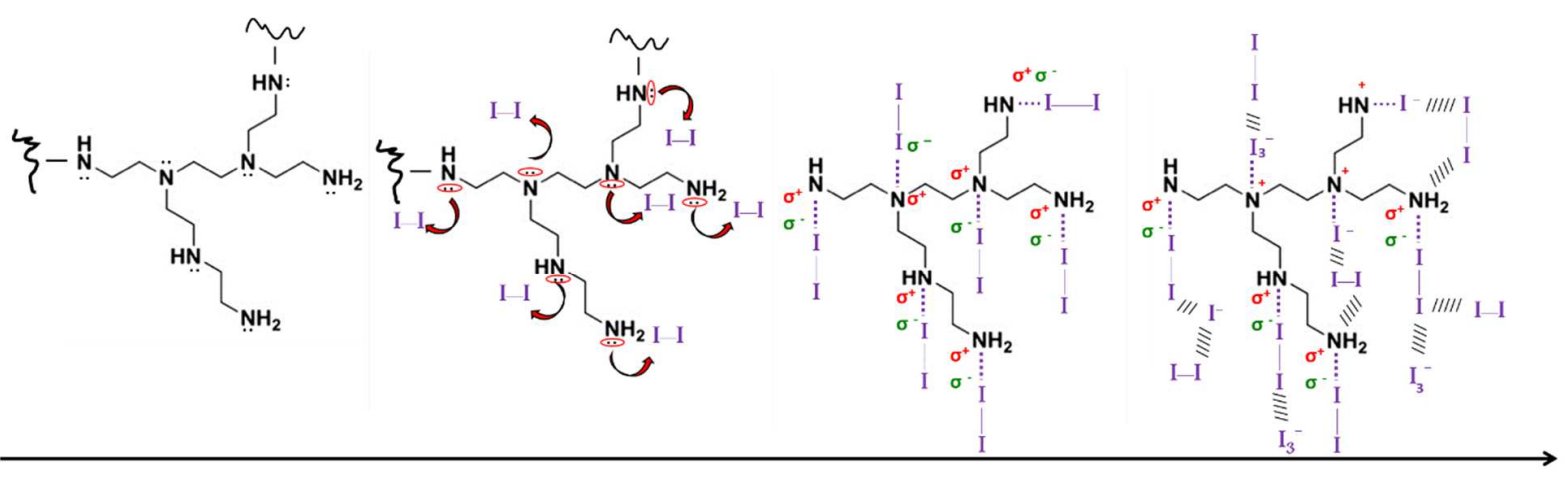

Addition of lodine $\left(I_{2}\right)$

\section{Conclusions}

In this work, the behavior of polyethyleneimine-impregnated nanoporous silicas was investigated for the first time in the context of iodine capture. First, the characterization of the chemical, textural and structural characteristics of SBA-15 sorbents was performed according to their PEI content (from 0 to $51 \mathrm{wt} \%$ ). It was found that the textural properties of SBA-15 quickly decreased due to the incorporation of increasing amounts of branched PEI oligomers in the pores or at the pore mouth. The post-grafting of an aminosilane compound before PEI impregnation did not seem to affect the dispersion of polyethyleneimine molecules in the pore system of SBA-15. Above $38 \mathrm{wt} \%$ of PEI, a significant fraction of PEI was even present as $a d$-layers on the external surface in an almost liquid-like state. This was confirmed by the features present in ATR/FTIR spectra or in TG curves, which were found to be somewhat closer to pure PEI for the highest PEI content $(51 \mathrm{wt} \%)$. In the case of PEI-impregnated Aerosil bearing no internal porosity, PEI liquid-like domains were located in the mesopores formed between silica nanoparticles. The thermal stability of the sorbents was found to be of the order of $200-250^{\circ} \mathrm{C}$ and did really strongly depend neither on the type of nanosilica used nor on the PEI content. 
The investigation of iodine adsorption behavior under liquid and gas-phase conditions at temperatures between 25 and $100^{\circ} \mathrm{C}$ revealed interesting features. The adsorption capacities were found to depend primarily on the $\mathrm{N}$ content of the sample due to the formation of specific halogen bonding between the amine moieties of PEI and iodine. Accordingly, the atomic $\mathrm{I}_{2} / \mathrm{N}$ ratio calculated for all the PEI-nanosilica sorbents were rather close to unity, except for the highest PEI content due to a limited accessibility of iodine to some adsorption sites. An exceptionally high adsorption capacity of more than $2 \mathrm{~g} / \mathrm{g}$ was measured for PEI-SBA-15 sorbent with $38 \mathrm{wt} \%$ PEI. This sorbent maintained its filtering properties even during a gas-phase at $100^{\circ} \mathrm{C}$ under flowing conditions. This value is above that reported earlier for the best silver zeolites and MOFs under similar testing conditions.

After-test characterization of the spent sorbents by TGA, DR-UV-Vis, Raman and FTIR spectroscopies revealed the co-existence of adsorbed ionic $\mathrm{I}_{\mathrm{n}}{ }^{-}$species together with complexed and physisorbed iodine. The formation of these different iodine adsorbed species is driven by charge-transfer phenomena and further reaction with water traces or silanol groups (in the case of ionic species).

\section{Competing interests}

The authors declare that they have no competing interests

\section{Acknowledgements}

This work is granted by the French ministry of research (MENRT). The experimental work in this research is also carried out in the frame of the Mire Project, managed by the French National Research Agency (ANR) and launched by IRSN. The authors also thank Pr. Cedric Carteret for useful discussions on FTIR. 


\section{Appendix A. Supplementary data}

Supplementary data related to this article are provided separately.

\section{REFERENCES}

[1] V. Saenko, V. Ivanov, A. Tsyb, T. Bogdanova, M. Tronko, Yu. Demidchik, S. Yamashita, Clin. Oncol. 23 (2011) 234-243.

[2] A. Hidaka, H. Yokoyama, J. Nucl. Sci. Technol. 54 (2017) 819-829.

[3] R.T. Jubin, P. Printed, C. Microfiche, R.T. Jubin, Consolidated Fuel Reprocessing Program a Literature Survey of Methods to Remove Iodine from Off-Gas Streams Using Solid Sorbents, Departement of Energy, Oak Ridge National Laboratory. Chemical technology Division, 1979.

[4] D. Haefner, Methods of Gas Phase Capture of Iodine from Fuel Reprocessing Off-Gas: A Literature Survey, 2007.

[5] W.P. Freeman, T.G. Mohacsi, J.L. Kovach, Evaluation of quaternary ammonium halides for removal of methyl iodide from flowing air streams, United States, 1985.

[6] B. Azambre, M. Chebbi, O. Leroy, L. Cantrel, Ind. Eng. Chem. Res. 57 (2018) 1468-1479.

[7] B. Azambre, M. Chebbi, ACS Appl. Mater. Interfaces. 9 (2017) 25194-25203.

[8] M. Chebbi, B. Azambre, L. Cantrel, M. Huvé, T. Albiol, Influence of structural, textural and chemical parameters of silver zeolites on the retention of methyl iodide, Microporous Mesoporous Mater. 244 (2017) 137-150.

[9] M. Chebbi, B. Azambre, L. Cantrel, A. Koch, J. Phys. Chem. C. 120 (2016) 18694-18706.

[10] D.F. Sava, M.A. Rodriguez, K.W. Chapman, P.J. Chupas, P.S. Crozier, T.M. Neno, J. Am. Chem. Soc. (2011) 4.

[11] B.J. Riley, J. Chun, J.V. Ryan, J. Matyáš, X.S. Li, D.W. Matson, S.K. Sundaram, D.M. Strachan, J.D. Vienna, RSC Adv. 1 (2011) 1704.

[12] C.-G. Lee, P.J.J. Alvarez, A. Nam, S.-J. Park, T. Do, U.-S. Choi, S.-H. Lee, J. Hazard. Mater. 325 (2017) 223-229.

[13] Y.S. Dzyazko, L.N. Ponomaryova, Y.M. Volfkovich, V.E. Sosenkin, V.N. Belyakov, Sep. Sci. Technol. 48 (2013) 2140-2149.

[14] N. Kononenko, V. Nikonenko, D. Grande, C. Larchet, L. Dammak, M. Fomenko, Yu. Volfkovich, Adv. Colloid Interface Sci. 246 (2017) 196-216.

[15] T.H. Bui, S.P. Hong, J. Yoon, Water Res. 134 (2018) 22-31.

[16] Y. Lan, M. Tong, Q. Yang, C. Zhong, CrystEngComm. 19 (2017) 4920-4926.

[17] X. Qian, B. Wang, Z.-Q. Zhu, H.-X. Sun, F. Ren, P. Mu, C. Ma, W.-D. Liang, A. Li, J. Hazard. Mater. 338 (2017) 224-232.

[18] B.J. Riley, J.D. Vienna, D.M. Strachan, J.S. McCloy, J.L. Jerden, J. Nucl. Mater. 470 (2016) 307-326.

[19] S.U. Nandanwar, K. Coldsnow, V. Utgikar, P. Sabharwall, D. Eric Aston, Chem. Eng. J. 306 (2016) 369-381.

[20] N. Mnasri, C. Charnay, L.-C. de Ménorval, Y. Moussaoui, E. Elaloui, J. Zajac, Microporous Mesoporous Mater. 196 (2014) 305-313.

[21] J.H. Yang, Y.-J. Cho, J.M. Shin, M.-S. Yim, J. Nucl. Mater. 465 (2015) 556-564.

[22] D. Zhao, Y. Wan, W. Zhou, Ordered Mesoporous Materials, 2012.

[23] D. Zhao, Science. 279 (1998) 548-552. 
[24] M. Chebbi, Piégeage d'espèces iodées volatiles sur des adsorbants poreux de type zéolithique dans le contexte d'un accident nucléaire grave, University of Lorraine, 2016.

[25] Y.S. Kim, A study on adsorption characteristics and deterioration patterns of an impregnated active carbon under a simulated service condition of the filteringsystem at a nuclear power plant, in: Proc. 20th DOENRC Nucl. Air Clean. Conf., Boston, Massachusetts (USA), 1988.

[26] J. Clayden, N. Greeves, S.G. Warren, Organic chemistry, 2nd ed, Oxford University Press, Oxford; New YorK, 2012.

[27] S. Kobayashi, Ethylenimine polymers, Prog. Polym. Sci. 15 (1990) 751-823.

[28] C. Chen, S.-T. Yang, W.-S. Ahn, R. Ryoo, Chem. Commun. (2009) 3627.

[29] A. Goeppert, M. Czaun, R.B. May, G.K.S. Prakash, G.A. Olah, S.R. Narayanan, J. Am. Chem. Soc. 133 (2011) 20164-20167.

[30] L.J. Gillespie, L.H.D. Fraser, J. Am. Chem. Soc. 58 (1936) 2260-2263.

[31] P. Larkin, Infrared and raman spectroscopy: principles and spectral interpretation, Elsevier, Amsterdam; Boston, 2011.

[32] X. Hou, L. Zhuang, B. Ma, S. Chen, H. He, F. Yin, Chem. Eng. Sci. 181 (2018) 315-325.

[33] K. Li, J. Jiang, S. Tian, F. Yan, X. Chen, J. Mater. Chem. A. 3 (2015) 2166-2175.

[34] X. Xu, C. Song, J.M. Andresen, B.G. Miller, A.W. Scaroni, Energy Fuels. 16 (2002) 14631469.

[35] P. Hashemi, M. Shamizadeh, A. Badiei, P.Z. Poor, A.R. Ghiasvand, A. Yarahmadi, Anal. Chim. Acta. 646 (2009) 1-5.

[36] K.S.W. Sing, Pure Appl. Chem. 57 (1985) 603-619.

[37] Z. Chen, S. Deng, H. Wei, B. Wang, J. Huang, G. Yu, ACS Appl. Mater. Interfaces. 5 (2013) 6937-6945.

[38] A. Heydari-Gorji, Y. Belmabkhout, A. Sayari, Langmuir. 27 (2011) 12411-12416.

[39] R. Sanz, G. Calleja, A. Arencibia, E.S. Sanz-Pérez, Energy Fuels. 27 (2013) 7637-7644.

[40] R. Sanz, G. Calleja, A. Arencibia, E.S. Sanz-Pérez, Appl. Surf. Sci. 256 (2010) 5323-5328.

[41] R.E. Buckles, J.P. Yuk, A.I. Popov, J. Am. Chem. Soc. 74 (1952) 4379-4381.

[42] W.C. Mann, INTERACTIONS OF HALOGENS WITH AMINES, Thames Polytechnic, University of Greenwich, 1974.

[43] P. Zakaria, J.P. Hutchinson, N. Avdalovic, Y. Liu, P.R. Haddad, Anal. Chem. 77 (2005) 417-423.

[44] R. Wu, L. Hu, F. Wang, M. Ye, H. Zou, J. Chromatogr. A. 1184 (2008) 369-392.

[45] M.E.A. Ibrahim, C.A. Lucy, Talanta. 100 (2012) 313-319.

[46] M. Takahashi, T. Hirano, S. Kitagawa, H. Ohtani, J. Chromatogr. A. 1232 (2012) 123-127.

[47] P. Deplano, F.A. Devillanova, J.R. Ferraro, F. Isaia, V. Lippolis, M.L. Appl. Spectrosc. 46 (1992) 1625-1629.

[48] H.T. Nguyen, D.D. Nguyen, J. Spanget-Larsen, Chem. Phys. Lett. 716 (2019) 119-125.

[49] T. Tassaing, M. Besnard, J. Phys. Chem. A. 101 (1997) 2803-2808.

[50] K. Unger, Adventures in Separation Science and Technology, ChemViews. (2016). 


\section{Graphical Abstract}

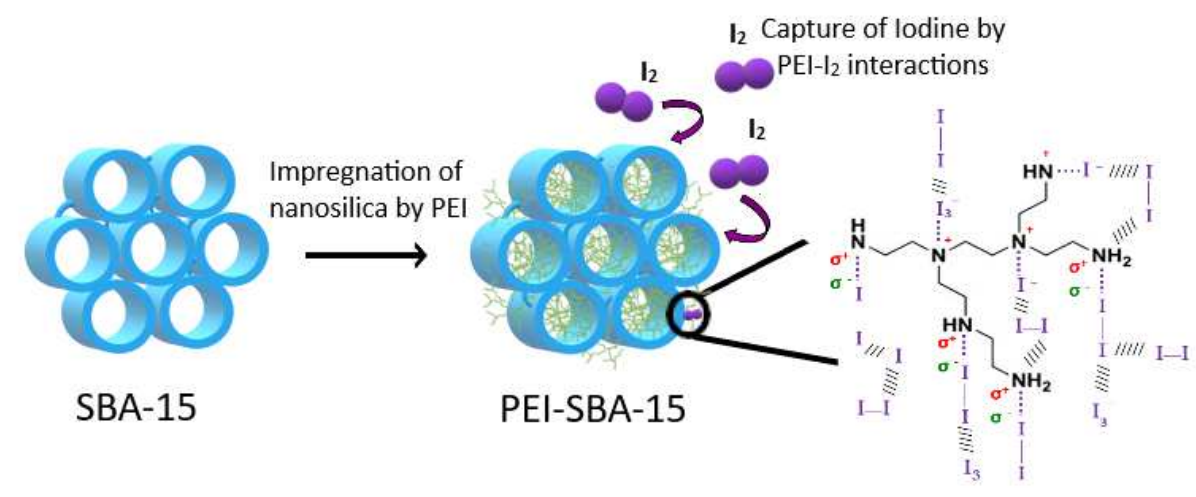

